\title{
Development of Advanced Actuators Using Shape Memory Alloys and Electrorheological Fluids
}

\section{Mavroidis}

Robotics and Mechatronics Laboratory, Department of Mechanical and Aerospace Engineering, Rutgers University, The State University of New Jersey, 98 Brett Road, Piscataway, NJ 08854-8058, USA

\begin{abstract}
Actuators are used to perform a variety of functions in almost every type of electromechanical system, "smart" device, and instrument. Increasingly, in many applications, actuators need to achieve reduced size, mass, power consumption, and cost. Examples of industries that demand novel, miniature, and powerful actuators are medicine, biotechnology, information technology, space, manufacturing, entertainment, military, and micro- and nanotechnology. Conventional actuators such as DC motors, pneumatic motors, and hydraulic motors are energy-wasting, largevolume, and heavy-mass actuation systems. Novel design methodologies, materials, and paradigms are currently needed in order to develop such lightweight and powerful actuation systems. In this paper we present the development of two novel, compact, and powerful smart material-based advanced actuators. The first motor is a shape memory alloy (SMA) bundle actuator, and the second is a hybrid concept based on electrorheological fluids (ERFs) and electromagnetic components. A detailed review of the state of the art in SMA- and ERF-based actuators is also presented.
\end{abstract}

\section{Introduction}

Robots, being an evolution of machines and mechanisms, originated by the ancient Greek, Alexandrian, and Roman engineers [1]. The first machines were simple jointed mechanisms that were actuated by human operators. The incorporation of an energy source, other than the human, to actuate and move certain components of the machine was a very important step toward automation of motion. Perhaps one of the earliest "roboticists" was the great Greek engineer and inventor Ctesibios (ca. 283-247 B.C.), who, applying knowledge of pneumatics and hydraulics, invented the precision clock. Heron of Alexandria (ca. first century A.D.), building on Ctesibios' work, wrote the fundamental textbook On Automatic Theaters, On Pneumatics and On Mechanics, which is considered the first well-documented robotic system description outside of mythology [2]. After this time, machines, mechanisms, and robotic technology evolved considerably during Roman and medieval times, the Renaissance, and the industrial revolution and modern era.

From the mechanical point of view, a robotic system is a mechanism composed of a set of links connected with joints that transfer motion and force from an input source to an output end-effector location. Different types of actuators have been used as input motion 
and force sources. Hydraulic, pneumatic, and electric actuators are called "conventional" because the majority of robotic and automated mechanical systems use them. Recently, advances in material technology have introduced lightweight and strong substances, making it possible to build structurally strong articulated mechanisms that are compact and weigh very little. Examples of such "smart" materials that can be used to develop novel actuators are shape memory alloys (SMAs), electrorheological fluids (ERFs), and electrostrictive and magnetostrictive materials including piezoelectric substances and electroactive polymers. Actuators made out of these smart materials are often called "nonconventional" to represent the novelty of the actuation concept. They are also called "advanced" to denote that they combine small, compact size with large force or velocity outputs.

There is a well-demonstrated need for commercial applications of advanced actuators. Current advances in information technology, nanotechnology, and biotechnology will require the development of miniaturized, novel devices and instruments that need to apply substantial forces. The same demand for new actuators occurs in traditional industries such as military, entertainment, medicine, and manufacturing, where there is an increasing need for developing small-size and lightweight devices able to apply large forces, develop high speeds, achieve large displacements, and be highly energy efficient. In all these applications, mission requirements are becoming drastically more stringent in terms of mass, dimensions, power, and cost. Improvements in actuator robustness and reliability associated with power efficiency and compact packaging can lead to effective devices that are significantly more capable and reliable at a lower cost.

Conventional actuators, such as electric motors and hydraulic and pneumatic cylinders, prevent large reductions in the overall weight and complexity of the robotic manipulator. Electric motors are heavy, and since they run at high speeds and produce low torques, they require a reduction gear system to produce the needed torques that are compatible with the motion of most mechanical devices. This increases the weight and complexity and has the added disadvantage of elevated noise and friction. Hydraulic and pneumatic actuators are much lighter than electric motors for the same power capability. However, they require a complex system of pumps, pressurized chambers, pipes, and valves to hold the working fluid. These systems are often noisy and prone to leaks, which are troublesome and messy at the least and hazardous at the most. They also have lower operation speed. Novel smart material-based advanced actuators can eliminate these disadvantages of conventional actuators. Detailed description of conventional actuators can be found in many robotics and haptics textbooks such as references [3, 4]. A very good comparative study of various actuator types in robotics can be found in reference [5].

To illustrate a comparison of the performance of various actuators, we present Fig. 1, which compares the power density versus the weight of conventional DC motors and hydraulic and pneumatic actuators to the corresponding characteristics of novel shape memory alloy (SMA) actuators and electrorheological fluid (ERF)-based motors called electrically controlled force and stiffness (ECFS) actuators (see Sec. 5 for a detailed presentation of ECFS actuators). The data for the ECFS actuators have been calculated using our preliminary results while the data for the other actuators have been found in reference [4]. From Fig. 1, it can be seen that hydraulic actuators can apply very large forces, but they weigh very much. DC motors are the weakest of the four types considered here, and their weight is small to moderate. SMAs are very strong (equivalent to hydraulic 


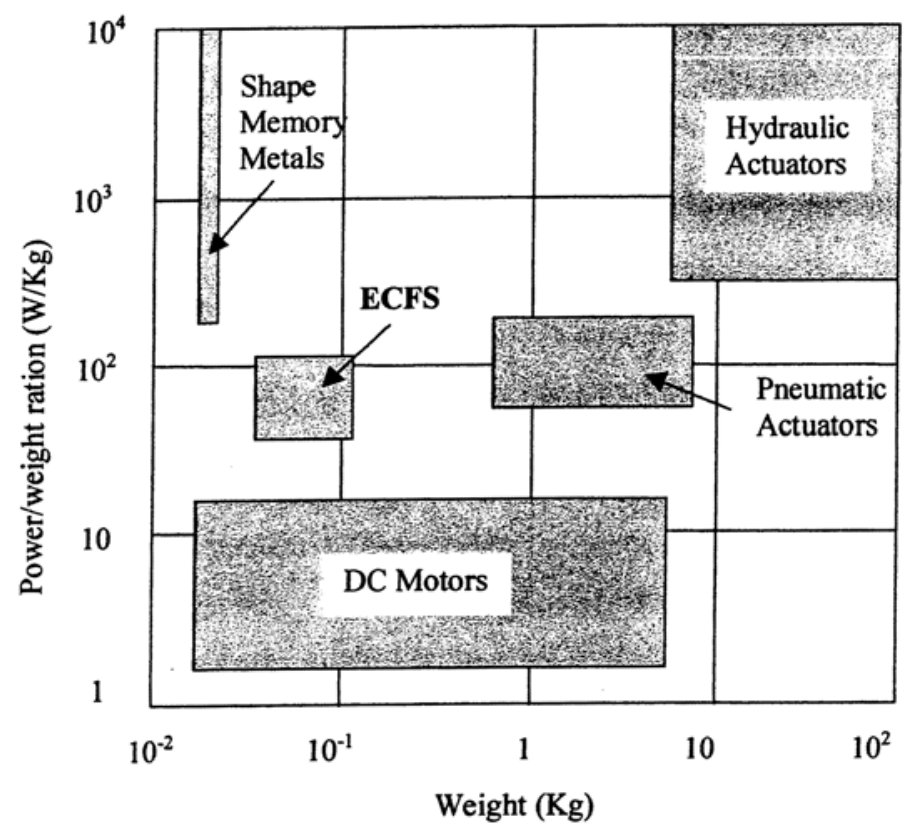

Fig. 1. Actuator comparison based on weight and output power-to-weight ratio.

actuators) and are incredibly small in size. Pneumatic and ECFS actuators can apply moderate forces, with the latter being much smaller in size. Figure 1 clearly demonstrates that smart material-based advanced actuators can reduce the size of actuators by at least two or three orders of magnitude while maintaining the same force-to-weight output.

The goal of the research performed at the Rutgers Robotics and Mechatronics Laboratory is to develop a new generation of actuation systems that are much lighter, simpler, and more compact than conventional actuation systems, but still retain the strength to perform the assigned task and are energy efficient. During the last four years we have developed several novel actuators using SMAs and ERFs. The objectives of this paper are (1) to present the principle of operation of SMAs and ERFs, (2) to review the research related to the design and control of SMA- and ERF-actuated devices, and (3) to present two novel advanced actuators, one based on SMAs and the other on ERFs. An initial version of this paper has been published in reference [6]. Here, we enhance our previous work with our latest results on the development of novel advanced actuators.

The structure of this paper is as follows. Section 2 presents the principles of operation of SMAs. Section 3 presents the design and control of a powerful and lightweight SMA bundle actuator that possesses impressive payload-lifting capabilities. The actuator consists of 48 nickel-titanium SMA wires mechanically bundled in parallel, forming one powerful artificial muscle. Section 4 discusses the fundamentals of ERFs. Finally, Sec. 5 describes the development of a novel ERF-based actuator called an electrically controlled force and stiffness (ECFS) actuator. 


\section{Shape Memory Alloy Actuators}

\subsection{History of SMA}

In 1932, a Swedish physicist by the name of Arne Olander discovered an interesting phenomenon when working with an alloy of gold $(\mathrm{Au})$ and cadmium $(\mathrm{Cd})$. The $\mathrm{Au}-$ $\mathrm{Cd}$ alloy could be plastically deformed when cool and then be heated to return to, or "remember," the original dimensional configuration. This phenomenon is known as the shape memory effect (SME), and the alloys that exhibit the behavior are called shape memory alloys (SMA). In 1958, researchers Chang and Read demonstrated the shape memory effect at the Brussels World's Fair. Specifically, they showed that the SME could be used to perform mechanical work by cyclically lifting a weight using a $\mathrm{Au}-\mathrm{Cd}$ SMA. Further research revealed other materials that demonstrate this phenomenon. In 1961, a group of U.S. Naval Ordnance Laboratory researchers led by William Beuhler stumbled across a significant discovery in the field of SME and SMA. While testing an alloy of nickel and titanium for heat and corrosion resistance, they found that it too exhibited the SME. The Ni-Ti SMA proved to be significantly less expensive, easier to work with, and less dangerous (from a health standpoint) than previously discovered alloys. These factors refreshed interest and research in the shape memory effect and its applications $[7,8]$.

Researchers, designers, and companies recognized the potential to use the SME in engineering applications. As a result, starting in the 1970s, commercial products began to appear. For the most part, the early devices functioned as fasteners and took advantage of a single shape memory dimensional change. Some examples of these static devices are couplings for piping systems and electrical connectors. Next, researchers began to propose SMA devices to perform dynamic tasks; thus, they began to play the role of actuators. In order to perform a dynamic task, the SMA must experience a cycle of heating, cooling, and deformation. This requirement led some companies, such as Delta Metal in England, to use SMA actuators in temperature regulation systems, where the environmental temperature could be used for thermal actuation. Delta Metal proposed that SMA devices could be used to automatically open and close greenhouse windows, operate valves that control building temperatures, and control automobile fan clutches. In 1982 Sharp incorporated SMA actuators into electric oven dampers, and in 1983 Matsushita Electric designed SMA-actuated louvers for air conditioners [7]. Other researchers pursued electricity (resistive or Joule heating) as a source of heat and thus actuation. In 1971, a team led by Sawyer developed and tested an artificial heart powered by electrical actuation of SMA elements. In 1983, Honma, Miwa, and Iguchi [9] showed that SMA actuation could be controlled by resistive heating and proposed that SMA actuators could be used in microrobotics. Research concerning the application and control of SMA actuators in robotic systems has continued and expanded through the present.

\subsection{Principle of Operation}

Shape memory alloys consist of a group of metallic materials that demonstrate the ability to return to some previously defined shape or size when subjected to the appropriate ther- 


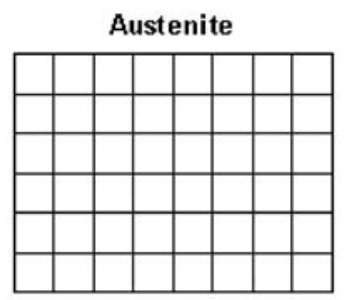

(a)

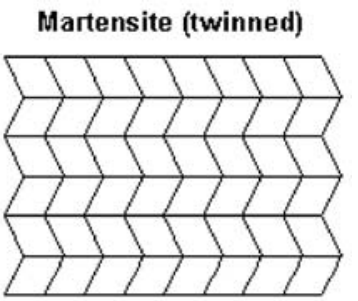

(b)

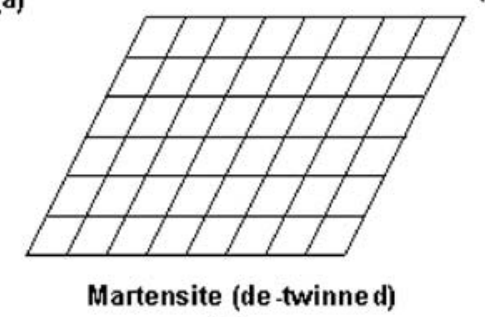

(c)

Fig. 2. Material crystalline arrangement during the shape memory effect.

mal procedure. Some examples of these alloys are $\mathrm{Ag}-\mathrm{Cd}, \mathrm{Au}-\mathrm{Cd}, \mathrm{Cu}-\mathrm{Al}-\mathrm{Ni}, \mathrm{Cu}-\mathrm{Sn}$, $\mathrm{Cu}-\mathrm{Zn}-(\mathrm{X}), \mathrm{In}-\mathrm{Ti}, \mathrm{Ni}-\mathrm{Al}, \mathrm{Ni}-\mathrm{Ti}, \mathrm{Fe}-\mathrm{Pt}, \mathrm{Mn}-\mathrm{Cu}$, and Fe-Mn-Si. The SME occurs due to a temperature- and stress-dependent shift in the material's crystalline structure between two different phases called martensite and austenite. Martensite, the low-temperature phase, is relatively soft, whereas austenite, the high-temperature phase, is relatively hard. For a simple example of the SME in action, consider the following. If a straight bar of some SMA in its austenitic (high-temperature) phase is allowed to cool below the phase-transition temperature, the crystalline structure will change to martensite. If the bar is subsequently plastically deformed, say, by bending, and then reheated above the phase-transition temperature, it will return to its original straight configuration. In order to understand this phenomenon, it is useful to consider the highly simplified twodimensional representation of the material's crystalline arrangement shown in Fig. 2.

Each box represents a grain of material with its corresponding grain boundaries. The grains form a heavily twinned structure, meaning that they are oriented symmetrically across grain boundaries. The twinned structure allows the internal lattice of individual grains to change while still maintaining the same interface with adjacent grains. As a result, shape memory alloys can experience large macroscopic deformations while maintaining remarkable order within the microscopic structure. For example, if a piece of SMA starts as austenite (Fig. 2a), the internal atomic lattice of each grain is cubic, creating grains with more or less right angles. If it is now allowed to cool below the phase-transition temperature, the crystalline structure changes to martensite (Fig. 2b) and the grains collapse to the structure represented by the diamonds. Note that the grains lean in different directions for different layers. Now, if sufficient stress is applied, the martensitic structure represented in Fig. 2b will start to yield and "de-twin" as the grains reorient such that they are all aligned in the same direction (see Fig. 2c). This behavior can be better understood by examining a typical stress-strain curve for the martensite phase (Fig. 3). For small stresses, the structure represented in Fig. $2 b$ behaves elastically 


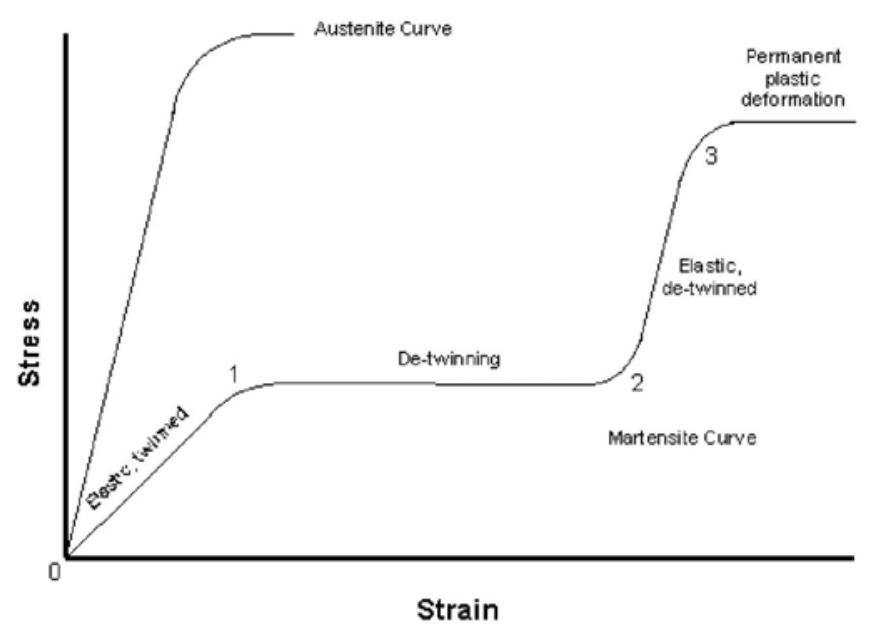

Fig. 3. Stress-strain relationship of a shape memory alloy.

(region 0 to 1). At 1, the material yields and de-twinning occurs between 1 and 2. At 2, the martensitic structure is entirely de- twinned as represented by Fig. 2c. Now, a second elastic region occurs from 2 to 3 . At 3 , permanent plastic deformation begins that is not recoverable by the SME.

The change that occurs within a SMA's crystalline structure during the SME is not a thermodynamically reversible process. In other words, there is energy dissipation due to internal friction and creation of structural defects. As a result, a temperature hysteresis occurs which is illustrated in Fig. 4a. Starting at 1, the material is 100\% martensite. During heating, the martensite/austenite composition follows the lower curve. When the temperature reaches $A_{S}$, austenite begins to form. Austenite continues to form until temperature $A_{F}$ is reached and the material is $100 \%$ austenite. If cooling occurs from 2 , the material composition follows the upper curve. When the temperature drops to $M_{S}$, martensite begins to form and continues to form until temperature $M_{F}$ is reached. Now the material is back to its starting condition-100\% martensite. This temperature hysteresis translates directly into hysteresis in the strain/temperature relationship (see Fig. 4b). The hysteresis behavior makes it challenging to develop modeling and control schemes for a SMA actuator. For a given SMA, the hysteresis is dependent on the composition of the alloy and the manufacturing processes. Most shape memory alloys have a hysteresis loop width of $10-50^{\circ} \mathrm{C}$, with the exception of some wide hysteresis alloys used for joining applications such as couplings [10]. Additional information on the principle of operation of SMAs can be found in references $[7,10]$.

\subsection{Nickel-Titanium (Ni-Ti) Shape Memory Alloy}

Of all the shape memory alloys that have been discovered so far, nickel-titanium (Ni-Ti) has proven to be the most flexible and beneficial in engineering applications. The following characteristics of Ni-Ti make it stand out from the other SMAs: greater ductility, 


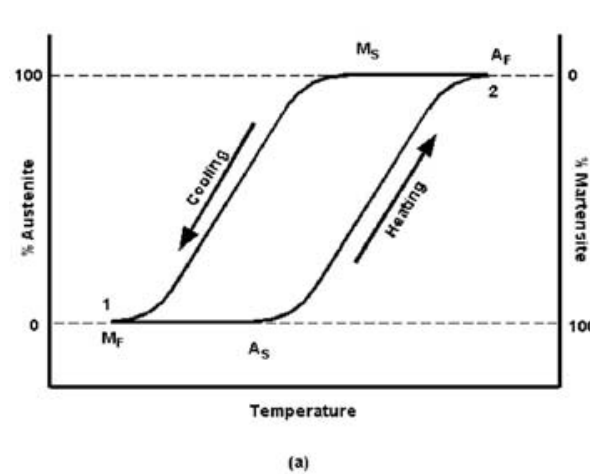

(a)

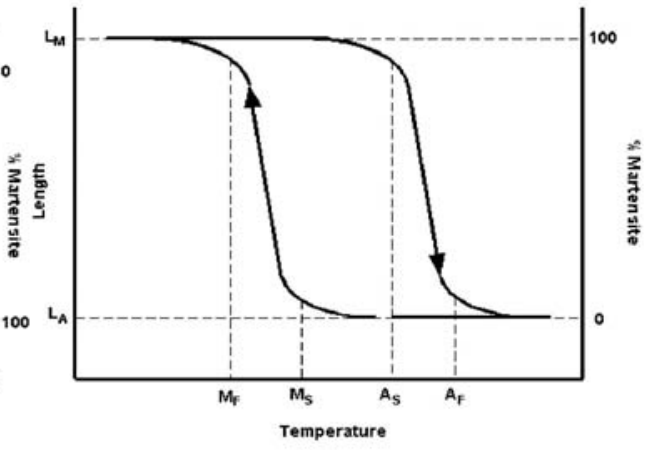

(b)

Fig. 4. Hysteresis loops in shape memory alloys.

more recoverable motion, excellent corrosion resistance (comparable to series 300 stainless steels), stable transformation temperatures, high biocompatability, and the ability to be electrically heated for shape recovery [10].

Ni-Ti SMA is the binary, equiatomic intermetallic compound of nickel and titanium. In simpler words, it is approximately 50 atomic $\% \mathrm{Ni}$ and 50 atomic\% Ti. The beneficial characteristics of the intermetallic compound are a moderate solubility range for excess $\mathrm{Ni}$ or $\mathrm{Ti}$, as well as for most other metallic elements, and a ductility comparable to most ordinary alloys. The solubility allows Ni-Ti to be alloyed with other elements to change the mechanical properties and phase-transformation temperature (here, phasetransformation temperature is taken to mean $A_{F}$ ). For example, adding additional $\mathrm{Ni}$ to the binary compound (up to $1 \%$ extra) strongly depresses the phase-transformation temperature and increases the yield strength of the austenite. Iron and chromium can also be added to lower the transformation temperature. By varying these and other elements, the transformation temperature can be varied from -200 to $110^{\circ} \mathrm{C}(-325$ to $230^{\circ} \mathrm{F}$ ). Copper can be added to decrease the hysteresis and lower the deformation stress (de-twinning stress) of the martensite. Table 1 shows the key physical properties of equiatomic Ni-Ti SMA [11].

Manufacturing Ni-Ti SMA and shaping it for a specific purpose is not a simple task. Since Ti is a very reactive element, melting must be done in an inert atmosphere. Common methods are plasma-arc melting, electron-beam melting, and vacuum-induction melting. Ni-Ti ingots can be initially shaped using standard hot-forming and cold-working processes. During cold-working the alloy work hardens very quickly and must be annealed frequently. Work hardening and the correct heat treatment can be used to improve the SMA's performance by reducing the stress needed to de-twin the martensite and increasing the strength in the austenite phase. Machining Ni-Ti through cutting methods is difficult, as is welding, brazing, and soldering. Grinding, shearing, and punching are often better methods to create specific shapes. The "memory configuration" of a SMA part is defined by restraining the part in the desired shape, and then heat treating at typically $500-800^{\circ} \mathrm{C}\left(950-1450^{\circ} \mathrm{F}\right)$ [11]. Companies such as Dynalloy, Inc., and Shape Memory Applications, Inc., provide prefabricated SMA elements such as wire, rod, rib- 
Table 1. Physical properties of Ni-Ti.

\begin{tabular}{|c|c|c|}
\hline Property & Austenite & Martensite \\
\hline Melting temperature, ${ }^{\circ} \mathbf{C}\left({ }^{\circ} \mathbf{F}\right)$ & \multicolumn{2}{|c|}{$1,300(2,370)$} \\
\hline Density, $\mathbf{g} / \mathrm{cm}^{3}\left(\mathbf{l b}_{m} / \mathbf{i n}^{3}{ }^{3}\right)$ & \multicolumn{2}{|c|}{$6.45(0.233)$} \\
\hline Resistivity, $\mu \Omega$-cm & Approx. 100 & Approx. 70 \\
\hline $\begin{array}{l}\text { Thermal Conductivity, } \mathrm{W}^{\circ} \mathrm{C} / \mathrm{cm} \\
\left(\text { Btu-hr- }^{-} \mathrm{F} / \mathrm{ft}\right)\end{array}$ & .18 (10) & $8.5(4.9)$ \\
\hline Corrosion resistance & \multicolumn{2}{|c|}{ Similar to 300 series stainless steel or titanium alloys } \\
\hline Young's modulus, GPa (1000 ksi) & Approx. 83 (12) & Approx. 28-41 (4-6) \\
\hline Yield strength, MPa (ksi) & $195-690(28-100)$ & $70-140(10-20)$ \\
\hline Ultimate tensile strength, MPa (ksi) & \multicolumn{2}{|c|}{$895(130)$} \\
\hline Transformation temperatures, ${ }^{\circ} \mathbf{C}\left({ }^{\circ} \mathbf{F}\right)$ & \multicolumn{2}{|c|}{-200 to $+110(-325$ to +230$)$} \\
\hline $\begin{array}{l}\text { Latent heat of transformation, } \\
\mathrm{kJ}-\mathrm{atom} / \mathrm{kg}(\text { cal-atom } / \mathrm{g})\end{array}$ & \multicolumn{2}{|c|}{$167(40)$} \\
\hline Shape memory strain & \multicolumn{2}{|c|}{$8.5 \%$ maximum } \\
\hline
\end{tabular}

bon, strip, sheet, and tubing. Additionally, they are able to create custom elements to user specifications.

\subsection{Shape Memory Alloy Actuators in Robotic Applications}

Using shape memory alloy actuators provides an interesting alternative to conventional actuation methods. Their advantages create a means to drastically reduce the size, weight, and complexity of robotic systems. First of all, SMA actuators possess an extremely high force-to-weight ratio. A Ni-Ti actuator can apply an actuation stress of $500 \mathrm{MPa}(72.5$ $\mathrm{ksi})$. So, a $150-\mu \mathrm{m}$-diameter $\mathrm{Ni}$-Ti wire can apply a force of $8.8 \mathrm{~N}$, which is $0.897 \mathrm{~kg}_{f}$ $\left(1.99 \mathrm{lb}_{f}\right)$. If the wire is $10 \mathrm{~cm}$ (3.94 in.) long, it will weigh $11.4 \mathrm{mg}\left(0.025 \mathrm{lb}_{m}\right)$ and can contract $0.85 \mathrm{~cm}$. So, the actuator can lift an object 78,000 times its own weight nearly $1 \mathrm{~cm}$ ! Granted, a simple electrical circuit is needed to heat the wire, but the forceto-weight ratio is still remarkable. Shape memory alloy actuators also are incredibly compact and simple. In the example described above, the actuator itself has a volume of only $0.002 \mathrm{~cm}^{2}$. A SMA actuation system consists only of the SMA element and a heating and cooling method. The cooling method can be as simple as a combination of natural convection, conduction, and radiation. A final advantage is noiseless operation. Whereas conventional actuators produce a significant amount of noise, the SMA actuator is completely silent.

Shape memory alloy actuators do have disadvantages, which must be thoroughly considered and analyzed prior to deciding to use SMA for an application. First of all, they operate with a low efficiency. A SMA actuator is effectively a heat engine in which the material converts thermal energy directly into work. Therefore, the efficiency of the actuator cannot be greater than that of the Carnot cycle. The efficiency of the Carnot cycle is low in the temperatures where typical SMA actuator operate-not exceeding $10 \%$ [12]. Second, SMA actuators operate at a low bandwidth, meaning that they are relative slow to cycle. The cycling time is primarily dependent on the heat transfer characteristics of the SMA "cooling system." The primary parameters that affect bandwidth are the 
temperature and type of surrounding medium, the convection of the surrounding medium, and the surface-to-volume ratio of the SMA elements. Depending on the environment, heat dissipation can be a problem. For a high-temperature, low-convection environment, the heat transfer to the surrounding medium is reduced, resulting in a lower bandwidth. For a low-temperature or high-convection environment, the heat transfer is improved and bandwidth is increased. However, greater heat transfer also means that more power is needed to achieve actuation temperature. Another disadvantage of SMA actuators is the small absolute strains achieved by the SMA material. With only $8.5 \%$ strain available (for Ni-Ti), mechanisms actuated by SMA that are required to create large motions must be cleverly designed. Converting small motions into large motions comes with the unavoidable reduction in mechanical advantage. A final disadvantage, and topic of much research, is the difficulty of controlling SMA actuators. The shape memory effect is a highly nonlinear phenomenon. Nonlinearities enter the process through the hysteresis behavior described earlier, nonlinear heat transfer, and any nonlinear change in the parameters that affects the phase composition of the material (temperature, stress). Another control issue is that the entire deflection of a SMA element occurs over a small temperature range, making accurate control in partial contraction difficult. Control is also difficult due to the structural elasticity of SMA actuators.

When designing a shape memory alloy actuator for a mechanism, one of the first decisions is specifying the source of heat to actuate the SMA element. In certain specialized applications, the temperature of the surrounding medium can be used as a source of heat. This method provides a excellent option when designing mechanisms that regulate temperature. For example, a SMA element can be placed in a medium (say air) whose temperature needs to be controlled. The SMA element can be manufactured such that its actuation temperature corresponds to some critical temperature of the medium. When the medium reaches the critical temperature, the SMA element actuates and possibly opens a valve to supply more cooling. Here, the SMA element acts as both the sensor and the actuator. No electronics are needed in this incredibly simple system.

For other applications, the typical source of heat to achieve actuation temperature is Joule heating by electrical current. The electrical source can be either DC or AC. If AC, it should be at a frequency significantly higher than the bandwidth of the SMA-actuated system, to avoid displacement fluctuations. The current $I$ that flows through a SMA element with resistance $R$, due to a certain voltage drop $V$ and the corresponding power $P$, can be found from the following well-known relationships:

$$
\begin{gathered}
I=\frac{V}{R}, \\
P=I V, \quad \text { or } \quad P=I^{2} R .
\end{gathered}
$$

Integrating a plot of power versus time and then dividing by the total time provides the average power. The average power required to achieve actuation temperature can be supplied by a steady or time-varying signal. An example of a time-varying signal that has been used extensively in electrical actuation is pulse-width modulation (PWM). The advantage of this method is more uniform heating of the SMA element. As expected, larger voltages/currents cause more rapid actuation. 


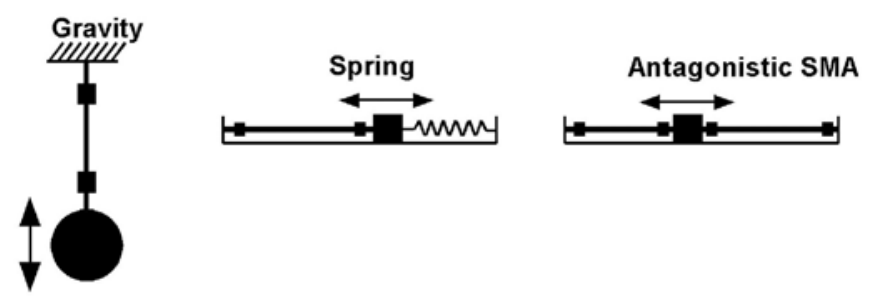

Fig. 5. Examples of bias forces in SMA actuated systems.

Shape memory alloy material can be formed into almost any shaped actuator imaginable. All that is needed is a heat treatment process to define the actuator's dimensional configuration in the austenite (actuated) phase. Some shapes that have been used are cantilever beams, wires, springs, ribbon, strip, sheet, and tubing. Although a SMA actuator could be designed such that it applies a force in three dimensions (depending on which direction it was deformed from the memory configuration), the great majority of SMA actuators apply a one-directional tensile force and cannot apply a compressive force directly. In order to apply a compressive force, the actuator dimensions would have to be large enough to ensure rigidity and prevent buckling. As discussed earlier, large dimensions would cause a major decrease in the surface-to-volume ratio and, consequently, bandwidth. The end result is that, in order to have a SMA actuator with sufficient bandwidth, the SMA element must be thin, making it only capable of applying tensile forces. Since most mechanisms require cyclic motions, a bias force is needed to return the mechanism in the opposite direction from which it was pulled by the SMA actuator. This bias force can be supplied by stored potential energy (gravity or a spring) or be provided by another SMA actuator working antagonistically. Simple examples of bias force are shown in Fig. 5. Some excellent basic design principals for single-element SMA Actuators can be found in reference [10].

The very simple mechanisms in Fig. 5 can achieve only small linear motions. In order to achieve large motions, a SMA actuator must be cleverly attached to the mechanism it operates. For a simple mechanism consisting of a moving link that pivots about a fixed revolute joint, the small linear displacements of a SMA actuator can be converted into large angular motions by fixing one end of the actuator and attaching the free end to the moving link close to the center of rotation of the revolute joint. This is very similar to the way biological muscles move the links that make up the body. Of course, mechanical advantage is lost as the free end of the actuator approaches the center of rotation. Figure 6 shows a schematic of one way to achieve large angular deflections from a SMA actuator.

If the SMA actuator pulls on a small, flexible cable that wraps around and fastens to a pulley fixed to the moving link, the relationship between the required pulley radius, $R_{p}$, the maximum SMA deflection, $\Delta_{\mathrm{SMA}}$, and the desired angular deflection of the moving link, $\Theta$, is

$$
R_{p}=\frac{\Delta_{\mathrm{SMA}}}{\Theta} .
$$

Clearly, from this relationship, for large angular deflections, $R_{p}$ must typically be small compared to the length of the moving link, $M$. Considering a static problem, the 

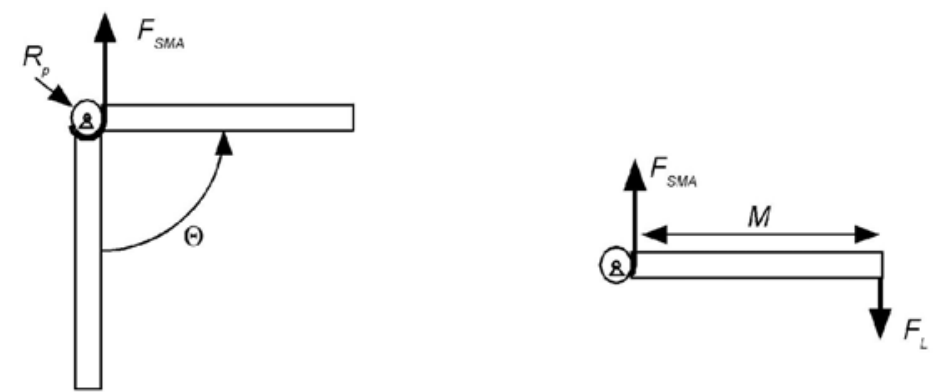

Fig. 6. Large angular deflections from a SMA actuator.

resulting ratio of required actuation force, $F_{\mathrm{SMA}}$, to the load, $F_{L}$, is

$$
\frac{F_{\mathrm{SMA}}}{F_{L}}=\frac{\Theta M}{\Delta_{\mathrm{SMA}}} .
$$

Hirose et al. [13] sought to improve the torque performace of this basic mechanism by using noncircular pulleys.

Equation (4) clearly shows that if SMA actuators are used in macrorobotic systems with revolute joints, the large angular motion requirement is satisfied by attaching the SMA actuator closer to the revolute joint axis. However, as shown in this equation, this attachment creates the need for large linear forces to be applied by the SMA actuators. It is clear that using thicker wires or connecting many wires mechanically in parallel in a "bundle" will increase the force capabilities of the SMA actuator. However, the actuator bandwidth and power supply requirements may be dramatically affected by such an arrangement and have to be taken into account when designing a SMA bundle actuator. This design problem motivated Mosley and Mavroidis $[14,15]$ to develop and test a SMA actuator that could apply large forces (up to $100 \mathrm{lb}_{f}$ ), retain an acceptable bandwidth, and use a reasonable power supply. This actuator is discussed in detail in Sec. 3.

\subsection{Modeling, Dynamics, and Control of Shape Memory Alloy Actuators}

Developing a mathematical model that captures the behavior of a shape memory alloy as it undergoes temperature, stress, and phase changes is a complicated and challenging problem. Researchers continue to study how best to model and control actuators that use this unique family of materials. As discussed earlier, it is the significant hysteresis loop that causes the problems. Gorbet and Wang [16] provide an excellent summary of SMA actuator modeling efforts to date. Some researchers have chosen to greatly simplify the material's behavior by creating dynamic models in which the phase-transition temperature is the same for heating and cooling, completely ignoring the effects of the large hysteresis. Kuribayashi [17] took the next step and developed a linear first-order model that estimates the hysteresis in the stress-strain behavior. 


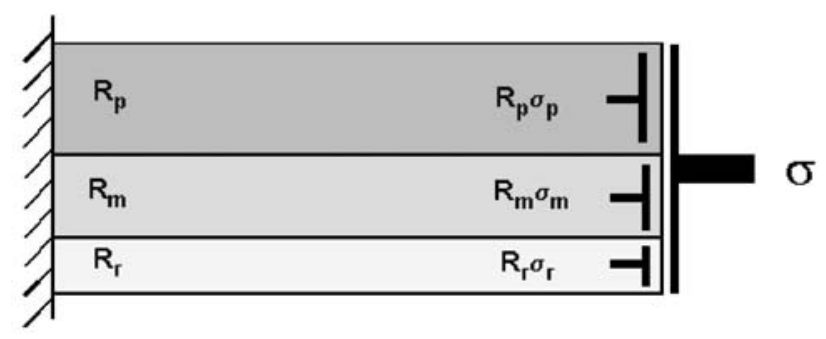

Fig. 7. A three-layer representation of a SMA.

To improve the accuracy, the model must be nonlinear. The complete model must capture the major hysteresis loop that occurs for changes from 100\% martensite to $100 \%$ austenite and vice versa, as well as the minor hysteresis loops that occur in between. Minor hysteresis loops are inherent in position control systems where heating and cooling cycles back and forth as the control system holds the actuator at the desired position. Ikuta et al. [18] developed a novel "variable sublayer model" in which, at a given time, the percentages of the different phases (including the intermediary Rhonbohedral phase) are described mathematically. As a result, for a given load, strain in the wire can be calculated from corresponding weightings of the respective strains of the different phases. Ikuta's work provided a basis for all nonlinear models to date. To understand Ikuta's modeling process fully, one should consult reference [18]. A basic description is provided below.

The "variable sublayer model" for Ni-Ti SMA is developed in three steps: (1) modeling the mechanical properties of the three independent phases; (2) modeling the thermoelastic transformation; and (3) combining steps 1 and 2, creating the "variable sublayer model." The material is modeled as three layers connected in parallel, representing the three different phases that can be present in the material at any given time (see Fig. 7).

The thickness of each layer corresponds to the volume fraction of the phase at a given moment. The total stress is a sum of the stresses in each layer weighted by the volume fraction. Resistivity of the material is modeled similar to stress-the total effective resistivity is the sum of the resistivities of each phase, each weighted by the volume fraction. Schematics of modeling the mechanical properties are shown in Fig. 8. The austenite phase (often referred to as the parent phase or P phase) is modeled as a simple elastic body. The martensite phase ( $\mathrm{M}$ phase) and the rhomohedral phase are both represented as a serial connection of an elastic and a plastic element. The thermoelastic transformation, including minor hysteresis loops, is modeled mathematically by a form of a "logistic curve" in which the equation gives the phase volume fraction as a function of temperature and stress. Ikuta compared his model to experimental results and showed greatly improved accuracy compared to previous models.

Madill and Wang [19] developed extensions to Ikuta's variable sublayer model that refined the modeling of the minor hysteresis loops, making it more suitable for dynamically modeling position control systems in which electricity is the source of heating. Their model consisted of two key elements, a temperature-current and a strain-temperature relationship. The temperature-current relationship was used to capture the dynamics of a Ni-Ti SMA actuator. The form of this relationship is a differential equation in terms 

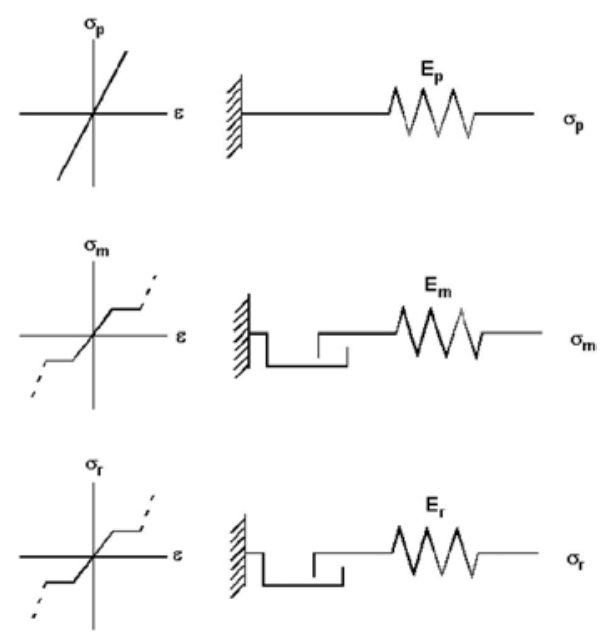

Fig. 8. Models of three phases.

of temperature, electrical current, and time:

$$
\rho c V \frac{d T}{d t}=R i^{2}(t)-h A\left[T(t)-T_{\infty}\right],
$$

where $\rho$ is the density of the SMA material, $c$ is the specific heat capacity of the SMA material, $V$ is the volume of the SMA material, $T$ is the wire temperature, $t$ is the time, $I$ is the current, $R$ is the electrical resistance, $h$ is the convection heat transfer coefficient, $A$ is the surface area of the SMA material, and $T$ is the ambient temperature.

The strain-temperature relationship, used to model hysteresis, involves two steps. First, temperature and stress must be related to phase composition. Second, phase composition must be related to strain. The resulting equations are somewhat lengthy, and one must consult reference [19] for a full explanation. The first step, relating temperature and stress to volume fraction of martensite, takes the following form:

$$
R_{m}(\theta ; t)= \begin{cases}\frac{R_{m a}^{C}(t)}{\left(1+e_{m}^{k_{m}^{C}\left(\theta-\beta^{C}\right)}\right)}+R_{m}^{C}(t), & \text { when cooling } \\ \frac{R_{m a}^{H}(t)}{\left(1+e^{k_{m}^{H}\left(\theta-\beta^{H}\right)}\right)}+R_{m b}^{H}(t), & \text { when heating }\end{cases}
$$

where $R_{m}$ is the martensite fraction, $\theta$ is the difference between the wire temperature $T$ and the ambient temperature $T_{\infty}, k_{m}$ is a temperature constant, $R_{m a}$ and $R_{m b}$ are constants that define the martensite fraction at the beginning and the end of the minor loops, and the superscripts $C$ and $H$ denote cooling and heating, respectively. Temperature and stress enter the equations in the exponential terms. The four functions, $R(t)$, are piecewise-constant functions of time which model the minor hysteresis loops. These functions remain constant during a period of heating or cooling. However, when a shift occurs from heating to cooling or vice versa, the functions change. Here is where the primary difference occurs between this model and that of Ikuta. Ikuta's modeling of 
minor hysteresis loops relied on empirically determining constant parameters for each minor loop, instead of time-varying functions, making it unsuitable for modeling closedloop control systems in which minor loops are occurring continuously. The second step, relating phase composition to strain, takes the following form:

$$
\varepsilon= \begin{cases}\frac{\sigma}{\left[E_{a}-\left(E_{a}-E_{m}\right) R_{m}\right]}, & 0 \leq \varepsilon<\varepsilon_{m}^{y} \\ \frac{\sigma+R_{m}\left(E_{T}-E_{m}\right) \varepsilon_{m}^{y}}{\left[E_{a}-\left(E_{a}-E_{T}\right) R_{m}\right]}, & \varepsilon_{m}^{y} \leq \varepsilon<\varepsilon_{m}^{d} \\ \frac{\sigma+R_{m}\left[\left(E_{T}-E_{m}\right) \varepsilon_{m}^{y}+\left(E_{d}-E_{T}\right) \varepsilon_{m}^{d}\right]}{\left[E_{a}-\left(E_{a}-E_{d}\right) R_{m}\right]}, & \varepsilon_{m}^{d}<\varepsilon\end{cases}
$$

where $\varepsilon$ is the tensile strain; $\sigma$ is the tensile load (MPa); $\sigma_{a}$ and $\sigma_{m}$ are stress (MPa) due to $100 \%$ austenite and martensite, respectively; $E_{a}, E_{m}, E_{T}$, and $E_{d}$ are the elasticity (MPa) of austenite, fully twinned martensite, partly twinned martensite, and detwinned martensite; $\varepsilon_{m}^{y}$ is the yield strain of twinned martensite; $\varepsilon_{m}^{d}$ is the minimum strain of detwinned martensite; and $R_{m}$ is the martensite fraction.

Madill and Wang [19] tested their model against results of both open- and closed-loop experiments. The correlation between the model and the experimental results was excellent for the open-loop testing as well as for closed-loop testing with a proportional gain controller. The correlation was very close for a PI-controlled closed-loop experiment.

The complexity of the SMA models described above makes it difficult to design controllers for SMA-actuated robotic systems mathematically. Despite the modeling difficulties, researchers have produced some very important results after applying several different controllers. Classical PID controllers have been studied by Reynaerts and Van Brussel [20]. Hashimoto et al. [21] applied a PD control scheme to the SMA wires used as actuators of a biped-walking robot. Ikuta et al. [22] used active PID control on a segmented active endoscope made with SMA springs. A PI controller including temperature feedback has been studied by Troisfontaine et al. [23]. Madill and Wang [19] used a very simple proportional control to verify their SMA system model and studied its stability. The control gains were tuned either on-line or through simulations with a trial-and-error method. The drawback of linear P, PI, or PID control is that the controller may perform well in the range where the control gains are tuned, but deteriorate dramatically once outside the range. Various adaptive control algorithms have been used on SMA actuators in order to compensate for the material's nonlinearities [24-26]. Grant and Hayward $[27,28]$ applied variable structure/sliding mode-control methods to perform position and force control tasks. Tebbe, Schroeder, and Butler [29] studied state-space multivariable control in large flexible smart structures using either eigenvalue pole placement or LQR methods.

\section{Design and Control of a SMA Bundle Actuator}

This section presents the design and control of a new and powerful Ni-Ti SMA-based actuator that can apply very large forces and thus can be used to operate the joints of macrorobotic mechanisms. Large forces are achieved by "bundling" a set of Ni-Ti wires, thus increasing the power of the actuator. A SMA bundle consisting of $48 \mathrm{Flexinol}^{\mathrm{TM}}$ 
wires was constructed and actuated by a computer-controlled electrical circuit. This actuator was designed to apply up to $100 \mathrm{lb}(445 \mathrm{~N})$, approximately 300 times its weight, over a maximum distance of $0.5 \mathrm{in} .(1.27 \mathrm{~cm})$. As far as the authors are aware, this is the first SMA wire bundle actuator with such force capabilities. In order to determine the performance characteristics of the SMA bundle, an instrumented test rig was designed and constructed. This setup was equipped with a load cell, linear displacement sensor, current and voltage sensors, and a thermocouple central to the bundle. Open- and closed-loop experiments were conducted on the SMA bundle with two different loading configurations, linear displacement and operation of a revolute joint. A PID-based controller with the addition of an input shaping function was developed for each loading configuration with excellent results, maintaining steady-state error within \pm 0.004 in. $(0.1 \mathrm{~mm})$ for linear motion and $\pm 1^{\circ}$ for revolute joint rotation. Here, the SMA bundle design and results of closed-loop control experiments are discussed. Results of the open-loop experiments are described in reference [14].

\subsection{Design Considerations}

In order to augment the force capabilities of SMA wire actuators, the overall crosssectional area of the SMA material normal to the direction of actuation must be increased. This can be achieved in two different ways-using thicker wires or connecting many wires mechanically in parallel. Although both of these methods achieve the goal of a more powerful actuator, there are other consequences that must be considered. Actuator bandwidth and power supply requirements are altered dramatically by changes in crosssectional area, and these effects must be taken into account.

One of the parameters that greatly affects the bandwidth of a Flexinol wire actuator is the diameter. The physical reason behind this behavior is the change in surface-to-volume ratio and thus the change in the heat transfer characteristics of the setup-the larger the surface-to-volume ratio, the greater the heat transfer, and the larger the bandwidth. So, in order to improve the force capabilities of a SMA actuator while not sacrificing bandwidth, it is more beneficial to use many thin wires connected mechanically in parallel rather than a single thick wire. Not only must thin wires be used, they also must remain separated so that the cooling medium, in this case air, can flow freely around all surfaces. One can think of this situation as analogous to the cooling tubes in a condenser, in which a large number of tubes are used to increase the heat transfer surface area. On the other hand, if many small wires were gathered together like the fibers in a rope, the advantages of the increased surface area would not be realized.

Initially, one may think that the best circuit design for the bundle itself is a parallel arrangement of the wires similar to how they are arranged mechanically. Although this is the simplest arrangement, it results in impractical power supply requirements, due to the low resistance. As an example, the effective resistance of a bundle consisting of fifty 12-in. (30.5-cm)-long, 0.006-in. (150- $\mu \mathrm{m}$ )-diameter wires all in parallel would be 0.305 $\Omega$. Given a required actuation current of $0.4 \mathrm{~A}$ through each wire $(20 \mathrm{~A}$ for the whole bundle), the resulting voltage drop across the bundle is $6.1 \mathrm{~V}$. (The required actuation current for each wire, $0.4 \mathrm{~A}$ in the case of a 0.006-in. Flexinol wire, is the current needed to cause complete contraction in $1 \mathrm{~s}$ when surrounded by room-temperature air.) 


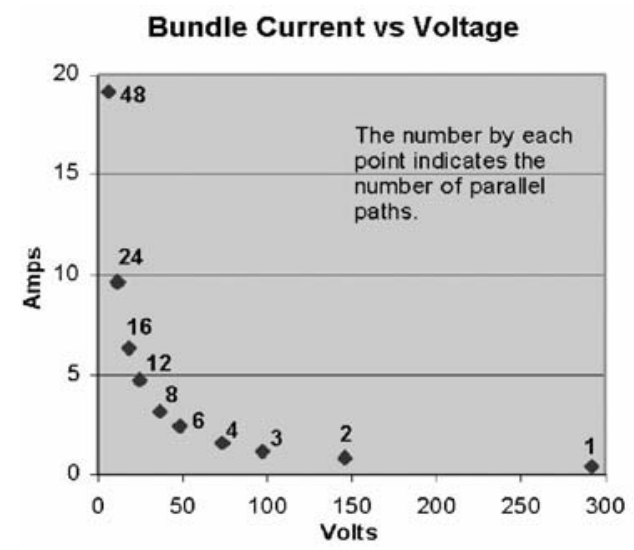

Fig. 9. SMA bundle current versus voltage.

This combination of low voltage and very high amperage can be avoided by creating a circuit in which the wires are arranged electrically in a combination of series and parallel paths while remaining mechanically connected in parallel. For a combination of wires in series and parallel,

$$
V_{B}=I_{\mathrm{SMA}} \cdot \frac{N}{P} \cdot L \cdot \bar{R} \quad \text { and } \quad I_{B}=P \cdot I_{\mathrm{SMA}}
$$

where $V_{B}$ and $I_{B}$ are the voltage drop across and current through the bundle, $I_{\mathrm{SMA}}$ is the single-wire actuation current, $N$ is the number of wires in the bundle, $P$ is the number of parallel paths, $L$ is the bundle length, and $\bar{R}$ is the single-wire linear resistance. Note that the ratio $N / P$ must be equal to an integer if identical paths are constructed. For the case where there are forty-eight 0.006-in. $(150-\mu \mathrm{m})$ wires, Fig. 9 shows a plot of current and voltage requirements in order to achieve actuation current $(0.4 \mathrm{~A})$ in each wire for different numbers of parallel paths.

In 1984, a SMA servoactuator was designed using four SMA elements mechanically connected in parallel and electrically connected in series [13]. The benefits of a lower current requirement, higher voltage requirement, and improved heat transfer over a single thicker wire were realized. However, if a large number of SMA elements, say 48, are connected completely in series (one parallel path), the required voltage for actuation becomes very large (see Fig. 9). Using different numbers of parallel paths allows the bundle to be tailored to different applications in which there may be current or voltage restrictions.

Considering the above discussion, SMA bundle design has four key parameters that determine the load capability, displacement capability, and current/voltage requirements. These parameters are diameter of the wire, the number of wires, the length of the bundle, and the number of parallel current paths. 


\subsection{SMA Bundle Experimental Setup}

The goal in this work was established to design an actuator that can apply a maximum of $100 \mathrm{lb}_{f}(445 \mathrm{~N})$ over a distance of approximately $0.5 \mathrm{in} .(1.27 \mathrm{~cm})$. A SMA bundle actuator with these capabilities will be able to power a revolute joint moving a lever arm that can lift and rotate approximately $5 \mathrm{lb}(2.3 \mathrm{~kg}$ ) of weight at the end of the arm through an angle of $90^{\circ}$. Demonstrating such payload and angular displacement capability from an SMA actuator will show that such actuators can be used effectively in macrorobotic applications.

Given that Flexinol wires can contract 5-8\% of their original dimension, a bundle length of 12 in. $(30.5 \mathrm{~cm})$ was chosen to meet the displacement criterion. Simple experiments were conducted to determine the weight-lifting capability of different diameter Flexinol wires. It was found that a single $0.006-i n .(150-\mu \mathrm{m})$ wire could lift over $2 \mathrm{lb}(0.9$ $\mathrm{kg}$ ) and possessed a sufficiently rapid cycling time for application in a prototype bundle. Considering that one wire can lift over $2 \mathrm{lb}$, in theory, fifty 0.006-in. wires connected mechanically in parallel could lift at least $100 \mathrm{lb}$. In the end, 48 wires were used, due to the symmetry of arrangement into a cylindrical bundle.

A SMA bundle was constructed consisting of forty-eight 12 -in. (30.5-cm)-long, 0.006in. $(150-\mu \mathrm{m})$-diameter wires $(N=48, L=12 \mathrm{in})$. The 48 wires were connected mechanically in parallel between two 0.25 -in. $(0.635$-cm)-thick, 1.5 -in. $(3.81-\mathrm{cm})$-diameter virgin Teflon ${ }^{\mathrm{TM}}$ end plates. Teflon was selected due to its high dielectric strength, temperature resistance, and good mechanical stability. Since all wires were not at the same voltage (series/parallel arrangement), it was necessary to keep each one electrically isolated from the others at the bundle end plates. Each wire passed through the end plate and was terminated with a 1/32-in. $(0.079-\mathrm{cm})$ copper crimp, providing an excellent mechanical and electrical connection (see Fig. 10). The copper crimps fit tightly into 48 sockets machined into each end plate.

In order to determine the performance characteristics of the SMA bundle, an experimental setup was designed and constructed (see Fig. 11). The experimental setup consisted of four main parts: (1) the test rig composed of the frame, the SMA bundle, the load, and various electrical power connections; (2) the power supply; (3) the control and instrumentation unit; and (4) a personal computer. The test rig frame provides a hanging point for the upper end of the SMA bundle. The lower/free end of the vertically oriented bundle is connected to the load. In the linear loading configuration, a variable weight and/or springs can act as the SMA bundle load.

In order to vary the number of parallel electrical paths through the SMA bundle, a "patch terminal" system was set up. The patch terminals consist of four 48-pin cannon plugs. A set of short patch wires was used to create the desired current path through the bundle. For all of the experiments conducted in this research, the patch terminals were wired for 8 parallel paths with 6 wires in series for each parallel path. As shown in Fig. 9, this results in a theoretical actuation voltage of $36.6 \mathrm{~V}$ and a current of $3.2 \mathrm{~A}$ for complete contraction of the bundle in $1 \mathrm{~s}$.

The power supply for bundle actuation was a Tellabs $48-\mathrm{V}$ (nominal), 10-A DC power source. The raw voltage from the power supply was controlled using a custom-designed operational amplifier circuit. The custom circuitry was based around a Burr Brown OPA 512 power operational amplifier. The gain of the amplifier was set to 10 so that a $0-5-\mathrm{V}$ 

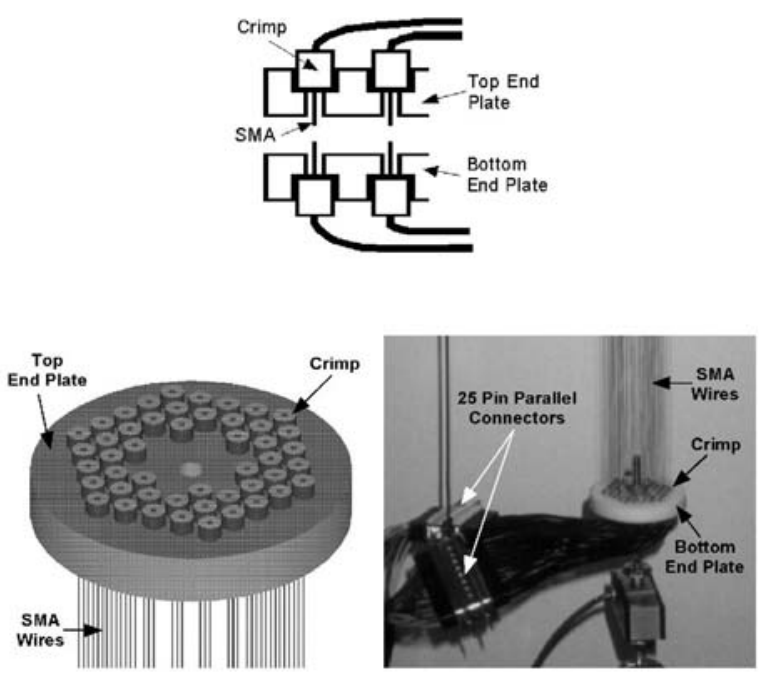

Fig. 10. Crimping schematic and bundle assembly.

input signal resulted in 0-50 V applied to the SMA bundle. A 350-MHz Dell PC was used for data acquisition and closed-loop control, running our Windows NT-based real-time control software named WinReC v.1.

\subsection{Closed-Loop Control}

Initially, a classical PID controller was developed to guide the SMA bundle as it lifted a weight. A set of step input responses for representative gains that showed good response are shown in Fig. 12. For this set of gains, the controller did not saturate, and thus the control voltage was a smooth function of time. Since there was no sharp change in control voltage, the rate of SMA bundle contraction changed smoothly and harmonic vibration was not initiated. For the typical response, the maximum steady-state error was approximately \pm 0.01 in. $( \pm 0.254 \mathrm{~mm})$. The responses shown in Fig. 12 were recorded in ideal environmental conditions with minimal disturbances. In a practical situation, changes in the temperature and velocity of the medium surrounding the bundle would result in thermal disturbances. Figure 13 displays the controller's ability to reject thermal disturbances created by rapidly waving a stiff piece of cardboard near the bundle for approximately $0.5 \mathrm{~s}$. The initiation of each disturbance is marked on the plot with arrows. Note the large position excursion that occurs due to the small, short-duration disturbance and that the controller takes over $2 \mathrm{~s}$ to correct the error. Mechanical disturbance tests were also run, showing the poor performance of this controller in rejecting mechanical disturbances. Although the controller presented above produced a smooth response to step inputs, it is clearly not suitable for high-accuracy, rapid tasks in a disturbance-filled environment. The controller gains need to be set higher for faster responses and better disturbance rejection. However, higher gains tend to saturate the controller during initial 

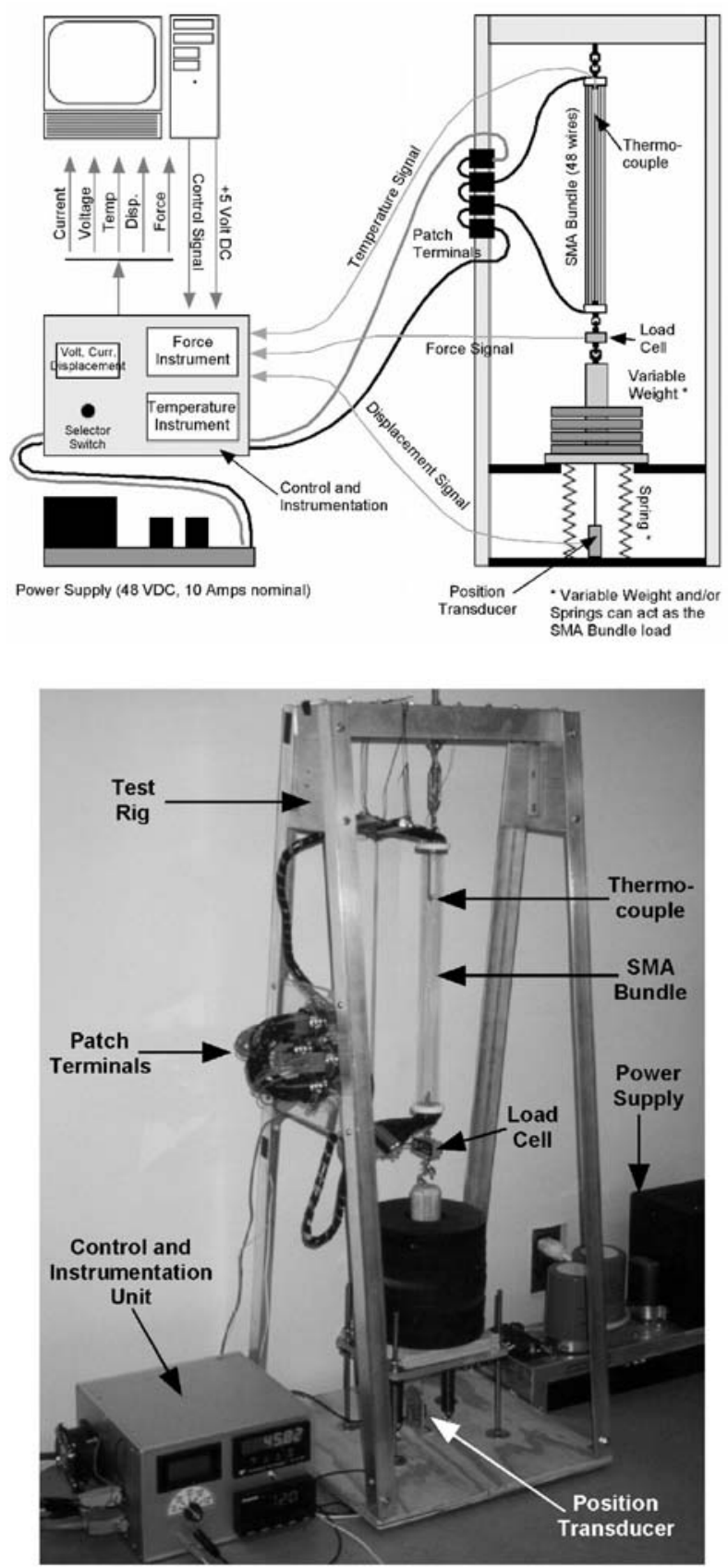

Fig. 11. SMA bundle experimental setup. 


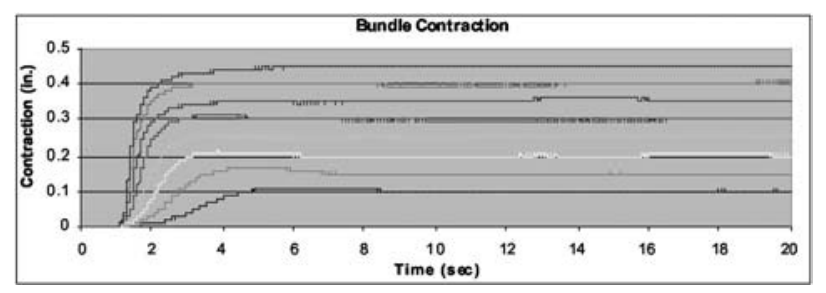

Fig. 12. Classical PID control of the SMA bundle.

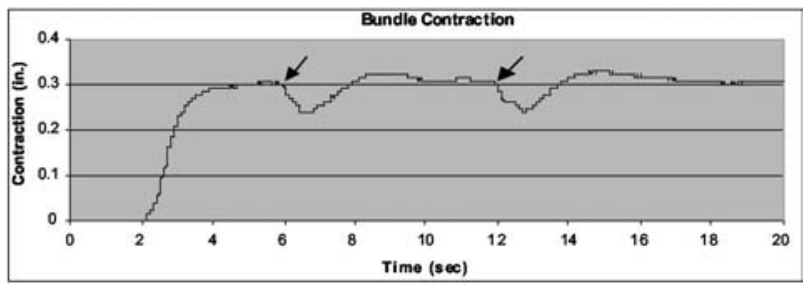

Fig. 13. Thermal disturbance rejection tests.

response to a step input and initiate harmonic vibration when the control voltage drops rapidly from saturation.

In order to eliminate the performance problems when higher-gain controllers are used, the output of the standard PID controller was shaped using an exponential function. The goal was to smooth the voltage transition when exiting or approaching the saturation limit. The exponential shaping function was used to (1) define the saturation limit when the control voltage was large and (2) approximate the control voltage when in the range of typical voltages needed to hold a certain position. The net effect was to reduce the gains for large control voltages and return the gains to near their original value when holding a certain position. An example of "shaping the control input" with this method is shown in Fig. 14. For the typical range of voltages needed to hold a certain position (10-20 V), the "exponential saturation" curve approximates the calculated control voltage. As the calculated control voltage gets higher, the "exponential saturation" curve approaches the desired saturation limit. When "exponential saturation" was applied to the PID controller, high gains could be set without generating large vibrations. Figure 15 shows the system responses to a set of step inputs. With higher gains, disturbance rejection was greatly improved. Figure 16 shows the controller rejecting four thermal disturbances of similar magnitude to the ones in Fig. 13. The errors are corrected in less than $1 \mathrm{~s}$.

\section{Electrorheological Fluids (ERF)}

\subsection{Introduction}

During the last 50 years, it has been known that there are liquids that respond mechanically to electrical stimulation. These liquids change their viscosity electroactively, and 


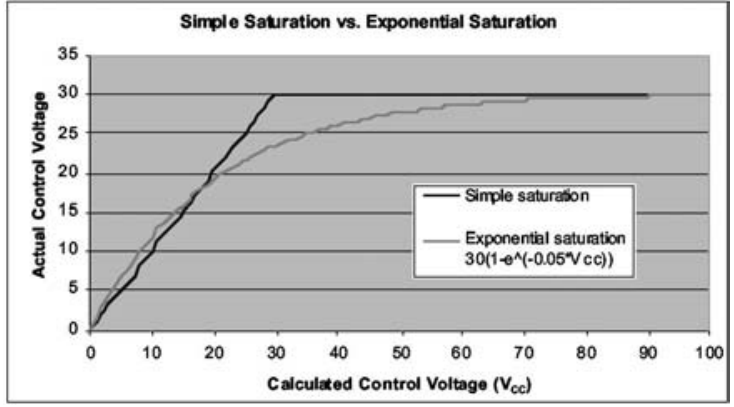

Fig. 14. Comparison of control inputs.

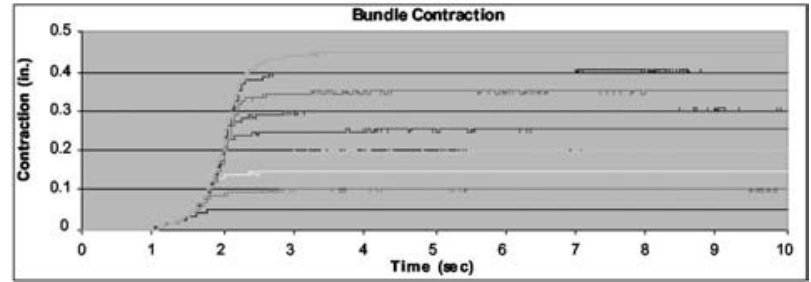

Fig. 15. Modified PID control of the SMA bundle.

they have attracted a great deal of interest of engineers and scientists. These electrorheological fluids (ERFs) exhibit a rapid, reversible, and tunable transition from a fluid state to a solidlike state upon the application of an external electric field [30]. Some of the advantages of ERFs are their high yield stress, low current density, and fast response (less than $1 \mathrm{~ms}$ ). ERFs can apply very high electrically controlled resistive forces, while their size (weight and geometric parameters) can be very small. Their long life and ability to function in a wide temperature range (as much as -40 to $+200^{\circ} \mathrm{C}$ ) allows for the possibility of their use in distant and extreme environments. ERFs are also not abrasive, nontoxic, and nonpolluting (meet health and safety regulations). ERFs can be combined with other actuator types such as electromagnetic, pneumatic, or electrochemical actuators so that novel, hybrid actuators are produced with high power density and low energy requirements. The electrically controlled rheological properties of ERFs can be beneficial to a wide range of technologies requiring damping or resistive force

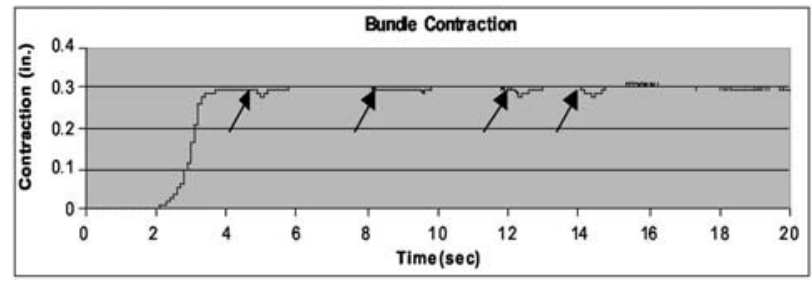

Fig. 16. Thermal disturbance rejection tests. 
generation. Examples of such applications are active vibration suppression and motion control. Several commercial applications have been explored, mostly in the automotive industry for ERF-based engine mounts, shock absorbers, clutches, and seat dampers. Other applications include variable-resistance exercise equipment, earthquake-resistant tall structures, and positioning devices [30].

While ERFs have fascinated scientists, engineers, and inventors for nearly 50 years, and have provided inspiration for developing ingenious machines and mechanisms, their applications in real-life problems and the commercialization of ERF-based devices has been very limited. There are several reasons for this. Due to the complexity and nonlinearities of their behavior, their closed-loop control is a difficult problem to solve. In addition, the need for high voltage to control ERF-based devices creates safety concerns for human operators, especially when ERFs are used in devices that will be in contact with humans. Their relatively high cost and the lack of a large variety of commercially available ERFs with different properties to satisfy various design specifications made the commercialization of ERF-based devices unprofitable. However, research on ERFs continues intensively, and new ERF-based devices are being proposed [31]. This gives rise to new technologies that can benefit from ERFs. One such new technological area is virtual reality and telepresence, enhanced with haptic (i.e., tactile and force) feedback systems and for use in, for example, medical applications.

\subsection{Principle of Operation}

Electrorheological fluids are fluids that experience dramatic changes in rheological properties, such as viscosity, in the presence of an electric field. Willis M. Winslow first explained the effect in the 1940s using oil dispersions of fine powders [32]. The fluids are made from suspensions of an insulating base fluid and particles of the order of $0.1-100 \mu \mathrm{m}$ in size. The volume fraction of the particles is between $20 \%$ and $60 \%$. The electrorheological effect, sometimes called the Winslow effect, is thought to arise from the difference in the dielectric constants of the fluid and particles. In the presence of an electric field, the particles, due to an induced dipole moment, will form chains along the field lines as shown in Fig. 17. The structure induced changes the ERF's viscosity, yield stress, and other properties, allowing the ERF to change consistency from that of a liquid to something that is viscoelastic, such as a gel, with response times to changes in electric fields of the order of milliseconds. Figure 18 shows the fluid state of an ERF without an applied electric field and the solidlike state (i.e., when an electric field is applied). Good reviews of the ERF phenomenon and the theoretical basis for ERF behavior can be found in references [33-36].

Under the influence of an electric field, the ERF alters its state from a Newtonian oil to a non-Newtonian Bingham plastic. As a Bingham plastic, the ERF exhibits a linear relationship between stress and strain rate like a Newtonian fluid, but only after a minimum required yield stress is exceeded. Before that point, it behaves as a solid. At stresses higher than this minimum yield stress, the fluid will flow, and the shear stress will continue to increase proportionally with the shear strain rate (see Fig. 19), so that

$$
\tau=\tau_{y}+\mu \dot{\gamma},
$$




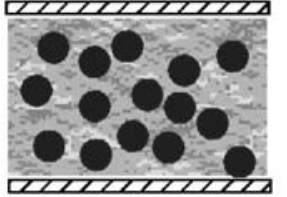

Without Field

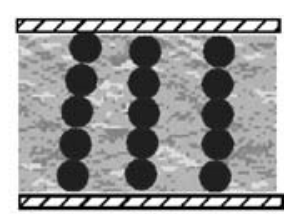

With Field

Fig. 17. Particle suspension forms chains when an electric field is applied.

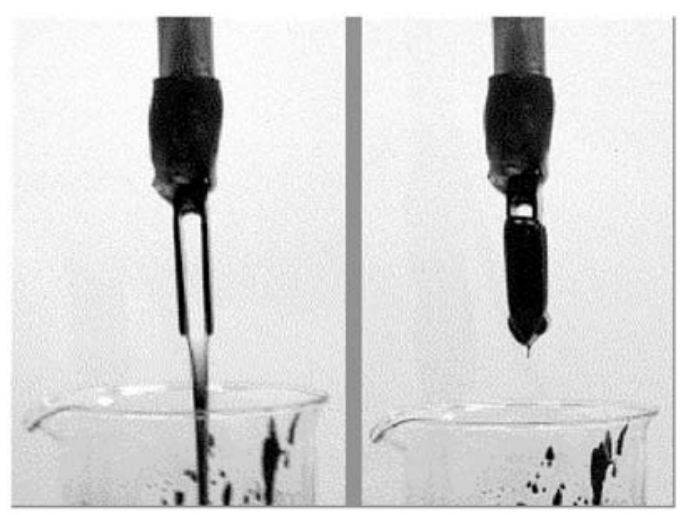

Fig. 18. Electrorheological fluid at reference (left) and activated states (right).

where $\tau$ is the shear stress, $\tau_{y}$ is the yield stress, $\mu$ is the dynamic viscosity, and $\gamma$ is the shear strain. The dot over the shear strain indicates its time derivative, the shear rate. An interesting phenomenon that has been observed by many researchers is that the dynamic viscosity becomes negative at high fields (see Fig. 19, reference [37]). This phenomenon can be explained by assuming that fewer, or weaker, bonds are formed at higher shear rates, thus giving a smaller total yield stress and the effect of negative dynamic viscosity [37].

Both the yield stress $\tau_{y}$ and the dynamic viscosity $\mu$ are two of the most important parameters that affect the design of ERF-based devices. The dynamic viscosity $\mu$ is mostly determined by the base fluid and the electric field. The field-induced yield stress $\tau_{y}$ depends on the electric field strength. For this dependence, some theoretical models have been derived, but none is yet able to reflect these relations properly. As a rule of thumb, one can assume that the yield stress increases quadratically with the electric field strength [38].

There are two important values for the yield stress: the static yield stress $\tau_{y, s}$ and the dynamic yield stress $\tau_{y, d}$. The static yield stress is defined as the value of stress needed to initiate flow, i.e., the stress needed to change from solid to liquid. The dynamic yield stress is the value of stress needed in zero-strain-rate conditions to go from a liquid to solid. Which one is larger is different from fluid to fluid. Most of the time, the static 


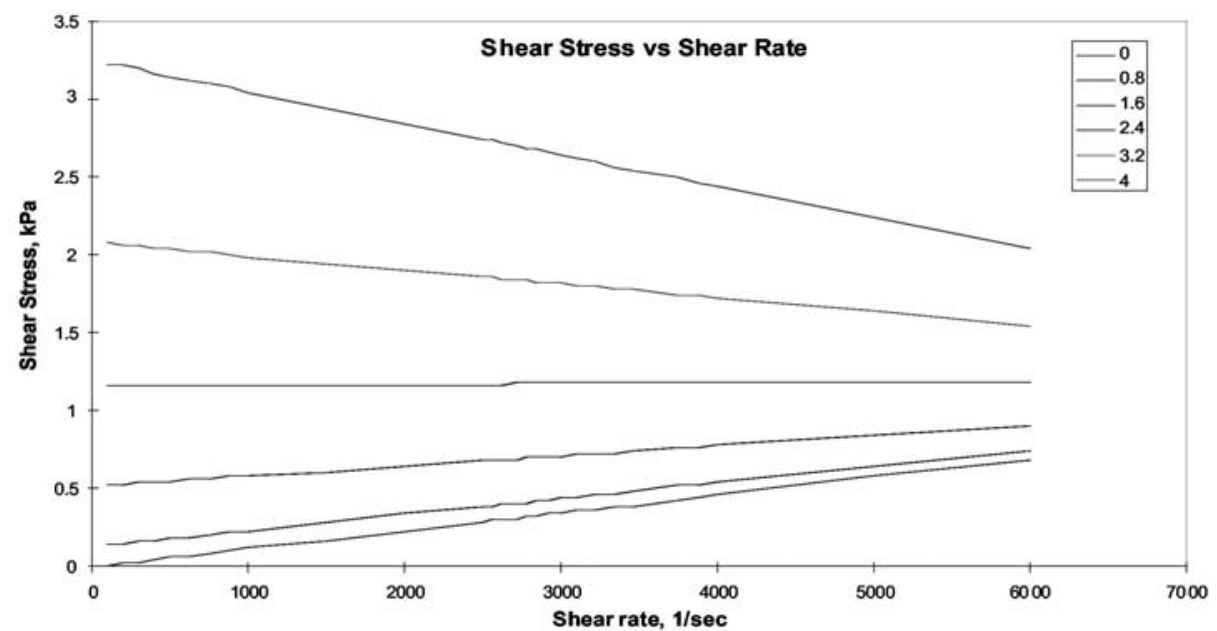

Fig. 19. Relationship between shear stress and shear strain rate in an ERF. (Reprinted by permission of ER Fluid Developments Ltd.)

yield stress is higher than the dynamic yield stress. This phenomenon, called "stiction," is highly dependent on the particle size and shape [35].

Another important parameter that needs to be known for ERFs is the current density $J$, defined as the current per unit electrode area. This parameter is needed to estimate the power consumption of ERF- based devices. Measurement of electric current through ERF materials is believed to be the result of charge leakage between particles [35].

ERF properties change with temperature, and this can tremendously affect the performance of ERF-based devices. "Good" ERFs should show constant properties over a large range of temperatures. There is no unified model describing the temperature dependence of the parameters of ERFs. This temperature dependence changes from fluid to fluid. The biggest temperature problem for ERFs results from the huge increase of current density with increasing temperatures. This increases power consumption but also increases safety concerns for human operators of ERF devices.

A good database of commercially available ERFs, including property comparison tables, can be found in reference [38]. A review of the material compositions for ERF patents can be found in reference [35]. As an example of ERFs we present here the electrorheological fluid LID 3354, manufactured by ER Fluid Developments Ltd. [37], which is used in the work presented in the next sections.

LID 3354 is an electrorheological fluid made up of $35 \%$ by volume of polymer particles in fluorosilicone base oil. It is designed for use as a general-purpose ER fluid with an optimal balance of critical properties. Its physical properties are density, $1.46 \times$ $10^{3} \mathrm{~kg} / \mathrm{m}^{3}$; viscosity, $125 \mathrm{mPa} . \mathrm{s}$ at $30^{\circ} \mathrm{C}$; boiling point, $>200^{\circ} \mathrm{C}$; flash point, $>150^{\circ} \mathrm{C}$; insoluble in water; freezing point, $<-20^{\circ} \mathrm{C}$. The field dependencies for this particular ERF are

$$
\tau_{y, s}=C_{s}\left(E-E_{\mathrm{ref}}\right), \quad \tau_{y, d}=C_{d} E^{2}, \quad \mu=\mu_{o}-C_{v} E^{2},
$$



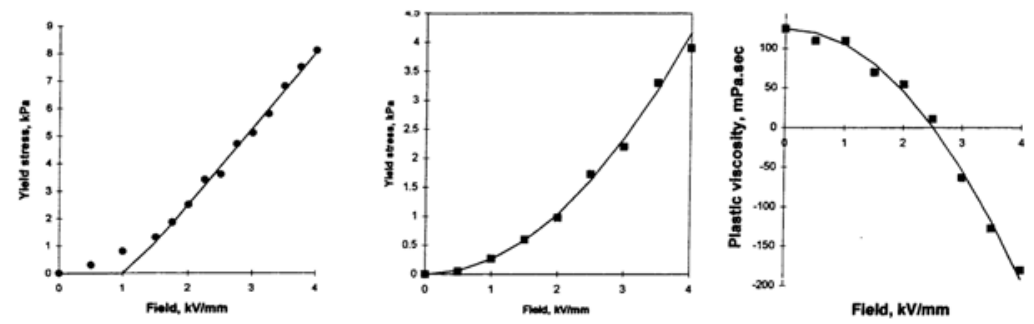

(a) Static Yield Stress at $30^{\circ} \mathrm{C}$

(b) Dynamic Yield Stress at $30^{\circ} \mathrm{C}$ (c) Plastic Viscosity at $30^{\circ} \mathrm{C}$

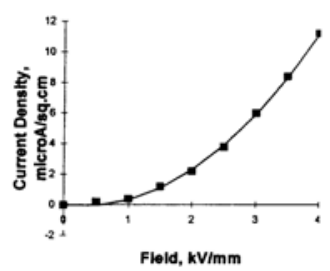

(d) Current Density at $30^{\circ} \mathrm{C}$

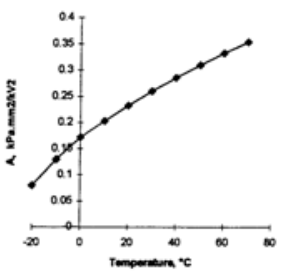

(e) Temperature Dependence of B,

Fig. 20. Technical information diagrams for the ER fluid LID 3354. (Reprinted by permission of ER Fluid Developments Ltd.)

where $\mu_{o}$ is the zero field viscosity; and $C_{s}, C_{d}, C_{v}$, and $E_{\text {ref }}$ are constants supplied by the manufacturer. The subscripts $s$ and $d$ correspond to the static and dynamic yield stresses, respectively. The formula for static yield stress is valid only for fields greater than $E_{\text {ref }}$. Figures $20 \mathrm{a}, \mathrm{b}$, and c are graphical representations of Eqs. (10) for the ERF LID 3354. Figure $20 \mathrm{~d}$ shows the dependence of the current density at $30^{\circ} \mathrm{C}$ as a function of the field. Figure 20e shows the coefficient $C_{d}$ of Eq. (10) as a function of temperature.

\subsection{Applications}

Control over a fluid's rheological properties offers the promise of many possibilities in engineering for actuation and control of mechanical motion. Devices that rely on hydraulics can benefit from ERFs' quick response times and reduction in device complexity. Their solidlike properties in the presence of a field can be used to transmit forces over a large range and have found a large number of applications. A good description of the engineering applications of ERFs can be found in references [39, 40]. Devices designed to utilize ERFs include engine mounts [41], active dampers and vibration suppression [42], clutches [43], brakes [44], and valves [40]. An important engineering application of ERFs is in vibration control, and a good review of the subject can be found in reference [45].

The application of ERFs in robotic has been very limited. They have been used mainly as active dampers for vibration suppression [46, 47]. Recently an ERF-based safety-oriented mechanism has been proposed for human-robot cooperative systems [48]. Kenaley and Cutkosky were the first to propose the use of ERFs for tactile sensing 
in robotic fingers [49]. Based on that work, several workers proposed the use of ERFs in tactile arrays used to interact with virtual environments [50] and also as assistive devices for the blind to read the Braille system. The first to propose this application of ERFs was Monkman [51]. Continuing this work, Taylor and his group at the University of Hull, UK, developed and tested experimentally a $5 \times 5$ ERF tactile array [52, 53]. Furusho and his group at Osaka University in Japan developed an ERF-based planar force- feedback manipulator system that interacts with a virtual environment [54, 55]. This system is actuated by low-inertia motors equipped with an ER clutch. An ERFbased force-feedback joystick has been developed at Fraunhofer-Institut in Germany. The joystick consists of a ball-and-socket joint in which an ERF has been placed in the space between the ball and the socket. The operator feels a resistive force to his or her motion resulting from the controlled viscosity of the ERF [56].

\section{Novel ERF-Based Elements and Actuators}

During the last two years Rutgers University and and the Jet Propulsion Laboratory have been involved in the development of novel ERF-based actuators and their application in haptic interfacing mechanisms that will enable a remote operator to "feel" the stiffness/damping and forces at remote or virtual sites [57-63]. The key aspects of the developed haptic system are miniature electrically controlled stiffness (ECS) elements and electrically controlled force and stiffness (ECFS) actuators that make use of ERFs. In this section we present the concept and some experimental results obtained from ECS elements and ECFS actuators.

\subsection{Electrically Controlled Stiffness (ECS) Elements}

The ECS element resistance to external loads is modified electrically by controlling the flow of an electrorheological fluid through slots on the side of a piston (Fig. 21). The ECS element consists of a piston that is designed to move inside a sealed cylinder filled with ERF. The rate of flow is controlled electrically by electrodes facing the flowing ERF while inside the channel. To control the "stiffness" of the ECS element, a voltage is applied between electrodes facing the slot, affecting the ability of the liquid to flow. Thus, the slot serves as a liquid valve, since the increased viscosity decreases the flow rate of the ERF and varies the stiffness felt. To increase the stiffness bandwidth from free flow to maximum viscosity, multiple slots are made along the piston surface. To wire such a piston to a power source, the piston and its shaft are made hollow and electric wires are connected to electrode plates mounted on the side of the slots. The inside surface of the ECS cylinder surrounding the piston is made of a metallic surface and serves as the ground and opposite polarity. A sleeve covers the piston shaft to protect it from dust, jamming, or obstruction. When a voltage is applied, potential is developed through the ERF along the piston channels, altering its viscosity. As a result of the increase in the ERF viscosity, the flow is slowed significantly, and resistance to external axial forces increases. 

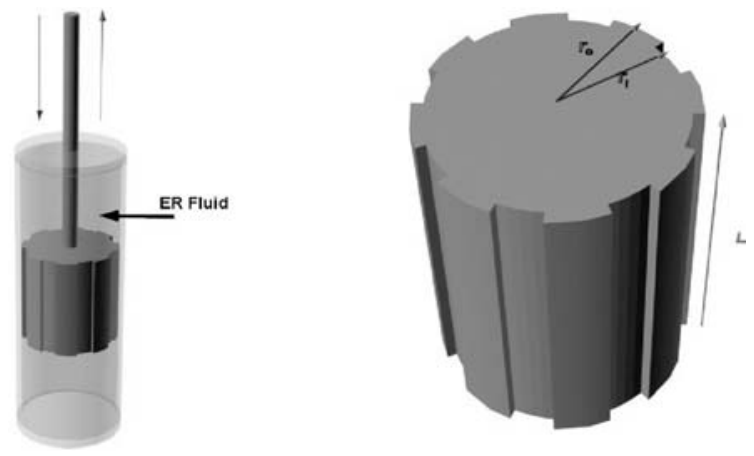

Fig. 21. ECS element and its piston.

To test the concept of controlling stiffness/damping with a miniature ECS element, a larger-scale testbed has been built at the Rutgers Robotics and Mechatronics Laboratory. This testbed, shown in Fig. 22, is equipped with temperature, pressure, force, and displacement sensors to monitor the ERF's state. Six system parameters are measured during experimentation: voltage, current, force, displacement, pressure, and temperature. All sensor signals are interfaced directly to analog-to-digital boards located in a Pentium II PC and are processed using the Rutgers WinRec v.1 real-time control and data acquisition Windows NT-based software. Representative results from tests are shown in Figs. 23a and b. In Fig. 23a no voltage is applied to the device. Four different weights equal to $2.75,5.50,8.25$, and $11 \mathrm{lb}$ are placed individually on the weight platform. A very fast descent of the piston is observed for all the weights. In Fig. 23b, the same procedure is followed, but this time a voltage of $2 \mathrm{kV}$ is applied on the ERF. It can clearly be seen that the piston is showing a very slow descent and for the lightest weight (i.e., $2.5 \mathrm{lb}$ ), no motion is observed. This experiment shows that when the electrical field is enabled, the viscosity of the ERF is such that the ECS element can resist the gravity forces from the weights.

\subsection{Electrically Controlled Force and Stiffness (ECFS) Actuators}

While the ECS element was able to resist externally applied forces in an electrically controlled way, it was not able to generate forces (i.e., to "push" back). Therefore, a new actuator concept was developed to be able to generate controlled forces and at the same time be able to control its compliance/damping electrically. The new actuators are called ECFS actuators.

The ECSF actuator is a small linear actuator that operates as an inchworm motor. Figure 24 shows a schematic of this inchworm motion. Motion is created through sequential activation of three elements. The outer two elements act as clamps or brakes on the output shaft. The central element, the mover, expands and contracts along the output shaft when voltage is applied. Though all three elements operate independently, they are physically connected. When a voltage is applied to the brake on the left, it clamps the shaft. Then a variable rate voltage is applied to the mover, causing it to expand. This 

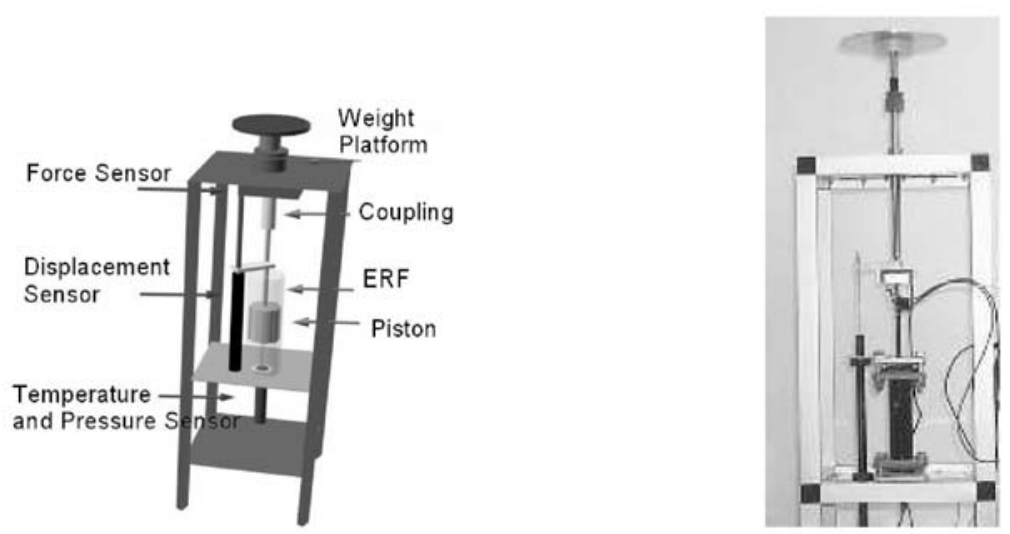

Fig. 22. ECS element experimental test bed.
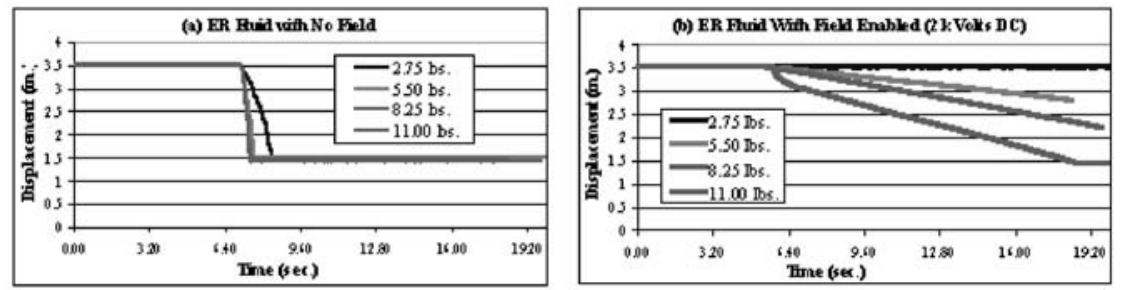

Fig. 23. Piston displacement.

motion is transferred to the output shaft that moves on the left. At the end of the motion of the mover element, a voltage is applied to the brake on the right, causing it to grip the shaft. Voltage is removed from the brake on the left, releasing it from the shaft. An opposite-sign voltage is applied to the mover, causing it to contract. When this motion is transferred to the shaft, it moves again on the left. This process is repeated as necessary, inching forward (or backward) as an inchworm does in nature.

In the ECFS actuators, the two brakes are ERF-based cylinders similar to our ECS concept. The mover element is composed of two electromagnetic cylinders that can attract and repulse themselves depending on the polarity of the current that passes through their windings. A schematic description of the ECFS actuator is shown in Fig. 25. The actuator consists of two pistons (brake elements) and two electromagnetic cylinders (mover element). Similar to the ECS element concept, each piston has several small channels with a fixed electrode plate. When an electric field is induced between the piston anode and cylinder cathode, the viscosity of the ERF increases and the flow rate of the fluid though the piston channel decreases, securing the piston to the cylinder wall.

Each of the electromagnetic cylinders consists of a coil and a ferromagnetic core integrated within the piston. When a current impulse is passed through the winding, an electromagnetic field is induced and, depending on the current direction, the cylinder moves forward or backward. This actuation principle is shown as a set of sequence diagrams in Fig. 26. In the first step, piston P1 is fixed relative to the cylinder by activating 


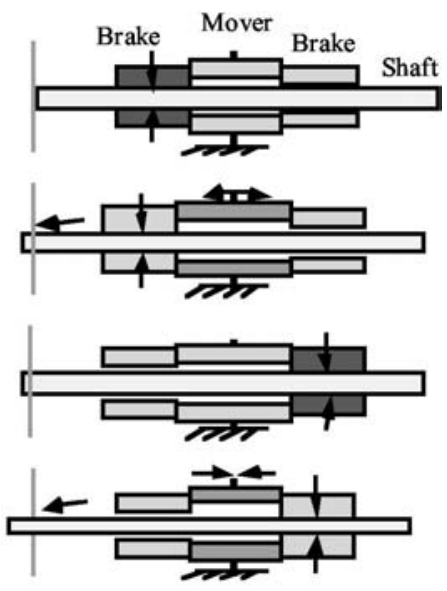

Fig. 24. Concept of the inchworm motor.

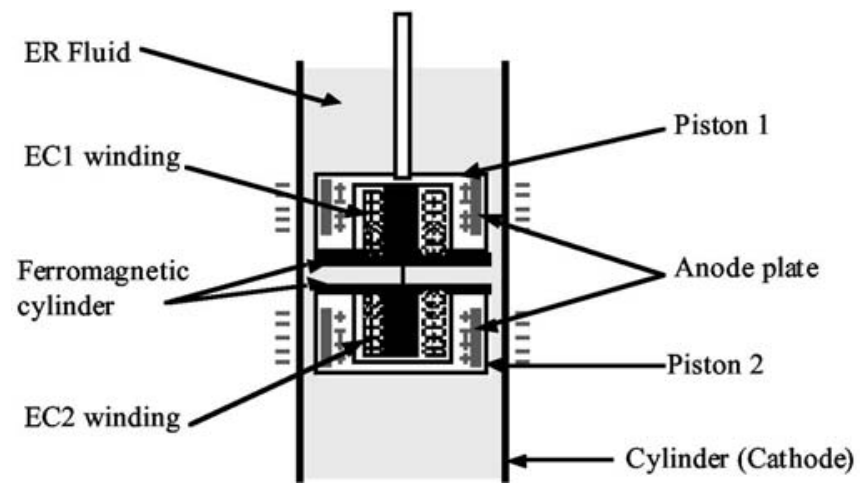

Fig. 25. ECFS actuator configuration.

its electrode and applying a voltage on the ERF; then, triggering the electromagnetic cylinder moves piston P2 forward. The ERF located between the two pistons is then displaced backward through the channels of piston P2. A horizontal channel is added at the surface of a ferromagnetic cylinder to increase the flow rate of the fluid. In the second step, the ERF in the channels of piston P2 is activated and P2 becomes fixed to the cylinder while P1 is disconnected from the cylinder. The current in the first winding is then reversed, changing the polarization of the magnetic cylinder, pushing P1 forward relative to P2. At each cycle, the pistons moves forward or backward with very small displacement $(<1.5 \mathrm{~mm})$. The duration of each cycle is close to a millisecond, corresponding to the response time of the ERF. The ECFS actuator can then reach a speed higher than $15 \mathrm{~cm} / \mathrm{s}$ with a piston displacement equal to $0.5 \mathrm{~mm}$ at $3-\mathrm{ms}$ cycle duration. The electromagnetic cylinder is designed to produce the same force as the resistive force of the piston inside the ERF, which is about $15 \mathrm{~N}$. 

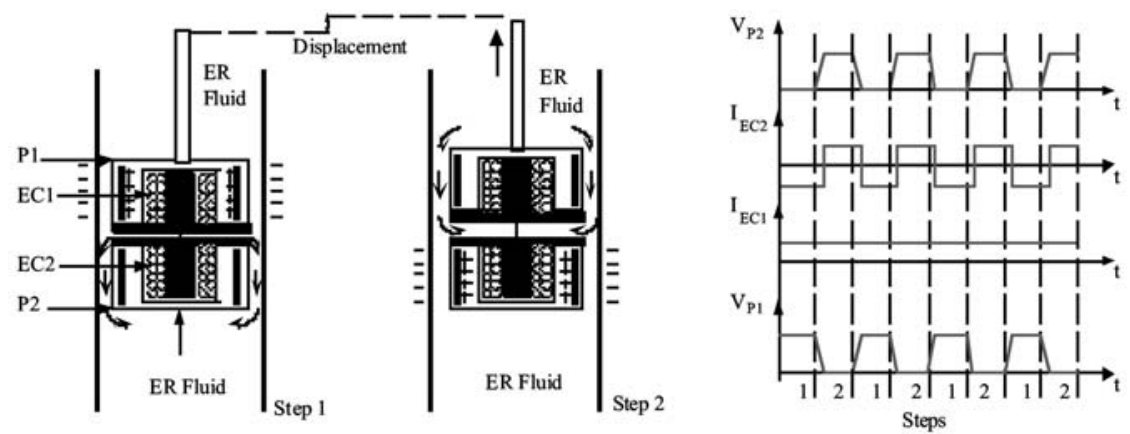

Fig. 26. Sequence diagram of ECFS actuators.

\section{Conclusions}

Using smart material-based advanced actuators in robotics can enable the development of many lightweight, compact, and strong systems. In this paper, we presented the development of shape memory alloy and electrorheological fluid-based actuators. The powerful, lightweight, and compact SMA wire bundle actuator designed, developed, tested, and controlled in this research provides a viable alternative to conventional actuators. It allows a method to drastically reduce the weight, complexity, and size of macrorobotic manipulators. The electrically controlled force and stiffness actuator that was also described uses electrorheological fluids to actively control the actuator compliance and damping while achieving high speeds and high resistive forces in very small sizes.

Acknowledgments. This work was supported by NASA's Jet Propulsion Laboratory (JPL), CAIP - the Center for Advanced Information Processing, and a Johnson and Johnson Discovery Award. The author would like to thank Mr. James Celestino, Ms. Kathryn DeLaurentis, Mr. Avi Fisch, Ms. Sandy Larios, Ms. Jamie Lennon, Mr. Michael Mosley, Mr. Alex Paljic, Mr. Charles Pfeiffer, and Ms. Sarah Young, from Rutgers University, for providing assistance during the development of the new actuator concepts. Special thanks and appreciation go to Dr. Yoseph Bar-Cohen of JPL and Dr. Mourad Bouzit of Rutgers University, who participated actively in the development of the ERF-based actuators. U.S. and PCT patent applications, filed by Rutgers University for the ERF-based actuators and elements, and for smart material-based advanced actuators for use in medical and rehabilitative devices, are currently pending.

\section{References}

1. A. Dimarogonas. In Modern Kinematics, Developments in the Last Forty Years, A. Erdman, ed. Wiley, New York (1993).

2. M. Rosheim. Robot Evolution: The Development of Anthrobotics. Wiley, New York (1994).

3. W. Stadler. Analytical Robotics and Mechatronics, McGraw-Hill, New York (1995).

4. G. Burdea. Force and Touch Feedback for Virtual Reality, Wiley, New York (1996).

5. J. Hollerbach, I. Hunter, and J. Ballantyne. In The Robotics Review 2, Q. Khatib, J. Craig, and LosanoPerez, eds., MIT Press, pp. 299-342. MIT Press, Cambridge, MA (1992). 
6. C. Mavroidis, C. Pfeiffer, and M. Mosley. In Automation, Miniature Robotics and Sensors for NonDestructive Testing and Evaluation, Y. Bar-Cohen, ed., pp. 189-214. American Society for Nondestructive Testing, (ASNT), (2000).

7. Toki Corporation. Biometal Guidebook. Toki Corporation, Tokyo (1987).

8. D. Grant. Shape Memory Alloy Actuator with an Application to a Robotic Eye, Thesis, McGill University, CA.

9. D. Honma, M. Yoshiyuki, and N. Igushi. Integrated Micro Motion Systems. Micro-machining, Control and Application, Nissin, Aichi, Japan, The 3rd Toyota Conference (1989).

10. T. Waram. Actuator Design Using Shape Memory Alloys, 2nd ed.

11. D. Hodgson, M. Wu, and R. Biermann. Shape Memory Alloys. http://www.sma-inc.com/SMAPaper.html (1998).

12. S. Hirose, K. Ikuta, and Y. Umetani. In Theory and Practice of Robots and Manipulators, Proc. RoManSy 1984, The Fifth CISM-IFToMM Symp. MIT Press, Cambridge, MA (1984).

13. S. Hirose, K. Ikuta, and K. Sato. Adv. Robotics 3(2):89-108 (1989).

14. M. Mosley and C. Mavroidis. J. Dynam. Syst. Measure. Control, Trans. ASME 123(1):103-112 (2001).

15. M. Mosley and C. Mavroidis. In Proc. 2000 ASME Mechanisms and Robotics Conf., Paper DETC2000/MECH-14157, Baltimore, MD (2000).

16. R. Gorbet and D. Wang. IEEE Trans. Control Syst. Technol. 6(4):554-562 (1998).

17. K. Kuribayashi. Int. J. Robotics Res. 4(4):47-58 (1986).

18. K. Ikuta, M. Tsukamoto, and S. Hirose. Proc. IEEE Micro Electromech. Syst. Invest. Microstruct, Sensors, Actuators, Machines, Robots 103-108 (1991).

19. D. Madill and D. Wang. IEEE Trans. Control Syst. Technol. 6(4):473-481 (1998).

20. D. Reynaerts and H. Van Brussel. In Proc. Fifth Int. Conf. on Advanced Robotics, New York, vol. 2, pp. 19-27 (1991).

21. M. Hashimoto, M. Takdeda, H. Sagawa, I. Chiba, and K. Sat. J. Robotic Syst. 2(1):3-25 (1985).

22. K. Ikuta, M. Tsukamoto, and S. Hirose. In Proc. 1988 IEEE Int. Conf. on Robotics and Automation, Philadelphia, vol. 1, pp. 427-430 (1988).

23. N. Troisfontaine, P. Bidaud, and P. Dario. In Proc. Experimental Robotics (ISER 97), Barcelona, Spain (1997).

24. C. A. Dickinson. Feedback Compensation of Shape Memory Alloy Hysteresis, Ph.D. thesis, Rensselaer Polytechnic Institute, Troy, NY (1997).

25. C. A. Dickinson and J. T. Wen. J. Intelligent Mater. Syst. Struct. 9(4):242-250 (1998).

26. G. Webb, L. Wilson, D. Lagoudas, and O. Rediniotis. AIAA J. 38(2):325-334 (2000).

27. D. Grant and V. Hayward. In Proc. 2000 IEEE Int. Conf. on Robotics and Automation, San Francisco, pp. 1314-1320 (2000).

28. D. Grant and V. Hayward. IEEE Control Syst. Mag. 17(3):80-88 (1997).

29. C. Tebbe, T. Schroeder, and R. Butler. Proc. SPIE-The International Society for Optical Engineering, Bellingham, WA, pp. 283-297 (1993).

30. P. Phule and J. Ginder. MRS Bull., 19-21 (August 1998).

31. R. Tao, ed. Proc. Seventh Int. Conf. on ER Fluids and MR Suspensions, Honolulu, Hawaii, World Scientific (1999).

32. W. M. Winslow. J. Appl. Phys. 20:1137 (1949).

33. H. Block and J. P. Kelly. J. Phys. D: Appl. Phys. 21:1661 (1988).

34. A. P. Gast and C. F. Zukoski. Adv. Colloid Interface Sci. 30:153 (1989).

35. K. D. Weiss, D. J. Carlson, and J. P. Coulter. In Advances in Intelligent Material Systems and StructuresVolume 2: Advances in Electrorheological Fluids, M. A. Kohudic , ed., pp. 30-52. Technomic, Lancaster, PA (1994).

36. H. Conrad. MRS Bull. 23(8):35-42 (1998).

37. ER Fluids Developments Ltd. Electro-Rheological Fluid LID 3354, Technical Information Sheet (1998).

38. D. Lampe. Materials Database on Commercially Available Electro- and Magnetorheological Fluids (ERF and MRF). http://www.tu-dresden.de/mwilr/lampe/HAUENG.HTM (updated 01/30/1997).

39. T. Duclos, J. Carlson, M. Chrzan, and J. P. Coulter. In Intelligent Structural Systems, Tzou and Anderson, eds., pp. 213-241. Kluwer, Netherlands.

40. J. P. Coulter, K. D. Weiss, and D. J. Carlson. In Advances in Intelligent Material Systems and StructuresVolume 2: Advances in Electrorheological Fluids, M. A. Kohudic, ed., pp. 64-75. Technomic, Lancaster, PA (1994). 
41. J. L. Sproston, R. Stanway, E. W. Williams, and S. Rigby. J. Electrostat. 32:253-259 (1994).

42. S.-B. Choi. Trans. ASME, J. Dynam. Syst., Measure. Control 121:134-138 (1999).

43. W. A. Bullough, A. R. Johnson, A. Hosseini-Sianaki, J. Makin, and R. Firoozian. Proc. Inst. Mech. Eng. I, J. Syst. Control Eng. 207(2):87-95 (1993).

44. M. Seed, G. S. Hobson, R. C. Tozer, and A. J. Simmonds. In Proc. IASTED Int. Symp. on Measurements, Processes and Controls, Sicily pp. 280-284 (1986).

45. R. Stanway, J. L. Sproston, and A. K. El-Wahed. Smart Mater. Struct. 5(4):464-482 (1996).

46. M. V. Ghandhi, B. S. Thompson, and S. Shakir. In Advances in Design Automation: Vol. 2; Robotics, Mechanisms and Machine Systems, ASME DE Vol. 10-2, pp. 1-10 (1987).

47. J. Furusho, G. Zhang, and M. Sakaguchi. In Proc. 1997 IEEE Int. Conf. on Robotics and Automation, pp. 3441-3448, Albuquerque, NM (1997).

48. F. Arai, K. Akiko, T. Fukuda, H. Matsuura, and H. Ota. In Proc. 1998 IEEE Int. Conf. on Robotics and Automation, Leuven, Belgium, pp. 2482-2487 (1998).

49. G. L. Kenaley and M. R. Cutkosky. In Proc. 1989 IEEE Int. Conf. on Robotics and Automation, Scottsdale AR, pp. 132-136 (1989).

50. D. Wood. Displays-Technol. Appl. 18(3):125-128 (1998).

51. G. J. Monkman. Presence 1(2) (1992).

52. P. M. Taylor, A. Hosseini-Sianaki, and C. J. Varley. In Proc. 1996 IEEE Int. Conf. on Robotics and Automation, Minneapolis, MN, pp. 18-23 (1996).

53. P. M. Taylor, A. Hosseini-Sianaki, and C. J. Varley. Int. J. Mod. Phys. B 10(23/24):3011-3018 (1996).

54. M. Sakaguchi and J. Furusho. In Proc. 1998 IEEE Int. Conf. on Robotics and Automation, Leuven, Belgium, pp. 2586-2590 (1998).

55. M. Sakaguchi and J. Furusho. In Proc. 1998 IEEE Virtual Reality Annual Int. Symp. (VRAIS), Atlanta, GA, pp. 66-70 (1998).

56. H. Böse, J. Berkemeier, and A. Trendler. In Proc. ACTUATOR 2000 Conf., Bremen, Germany (2000).

57. Y. Bar-Cohen, C. Pfeiffer, C. Mavroidis, and B. Dolgin. NASA Tech. Briefs 24(2):7a-7b (2000).

58. Y. Bar-Cohen, C. Mavroidis, M. Bouzit, B. Dolgin, D. Harm, G. Kopchok, R. White. Smart-Systems 2000: The International Conference for Smart Systems and Robotics for Medicine and Space Applications, Houston, TX (2000).

59. Y. Bar-Cohen, C. Mavroidis, C. Pfeiffer, C. Culbert, and D. Magruder. In Automation, Miniature Robotics and Sensors for Non-destructive Testing and Evaluation, Y. Bar-Cohen, ed., pp. 461-468. American Society for Nondestructive Testing, (ASNT) (2000).

60. Y. Bar-Cohen, C. Mavroidis, M. Bouzit, C. Pfeiffer, and B. Dolgin. Remote Mechanical Mirroring Using Controlled Stiffness and Actuators (MEMICA), Rutgers Docket Number 99-0056. U.S. and International PCT patent application filed by Rutgers University (2000).

61. C. Mavroidis, C. Pfeiffer, and Y. Bar-Cohen. In Proc. 2000 SPIE Conf. on Electro-Active Polymer Actuators and Devices (EAPAD 2000), SPIE 7th Annual Int. Symp. on Smart Materials and Structures, Newport Beach, CA, vol. 3987, pp. 300-310 (2000).

62. C. Mavroidis, C. Pfeiffer, J. Celestino, and Y. Bar-Cohen. In Proc. 2000 ASME Mechanisms and Robotics Conf., Baltimore MD, Paper DETC2000/MECH-14121 (2000).

63. C. Mavroidis, C. Pfeiffer, J. Lennon, A. Paljic, J. Celestino, and Y. Bar-Cohen. In Proc. ROBOTICS 2000 Conf:: The 4th Int. Conf. and Exposition/Demonstration on Robotics for Challenging Situations and Environments, Albuquerque, NM, pp. 174-180 (2000). 